\title{
DECOMPOSITION THEORIES FOR MODULES
}

BY

JOE W. FISHER( $\left.{ }^{1}\right)$

Introduction. Lesieur and Croisot in [7] have generalized the classical primary decomposition theory for Noetherian modules over commutative rings to the tertiary decomposition theory for Noetherian modules over rings, which are not necessarily commutative, but which have a certain chain condition on ideals. Riley has shown in [8] that for finitely generated unitary modules over left Noetherian rings with identities, the tertiary decomposition theory-in a certain sense-is the only natural generalization of the classical primary theory. The purpose of this paper is to show that the tertiary decomposition theory extends to a larger family of modules. We call this family the family of $t$-worthy modules. In particular we show that for an arbitrary ring $R$, the tertiary theory holds for any $R$-module $M$ which has the property that each factor module of $M$ is finite dimensional in the sense of Goldie. For the family of $t$-worthy modules we show that, with certain reasonable assumptions, the tertiary theory is the only theory that provides all the salient features of an ideal theory in the sense of the classical primary theory.

In order to make this extension, we introduce the abstract concepts of radical functions, associated ideal functions, and decomposition theories. From a radical function we construct a decomposition theory on a certain family of modules. Moreover we show that any decomposition theory can be constructed in this way from a radical function. Therefore our technique appears to be the natural way to construct decomposition theories. Hence we proceed by constructing decomposition theories in this way from various radical functions.

In $\$ 5$ we construct a decomposition theory from the tertiary radical function, $t$, and prove that it is the tertiary decomposition theory. Moreover we show that a necessary and sufficient condition for the tertiary decomposition theory to exist on an admissible family $C$ of modules is that each module in $C$ be $t$-worthy. Goldie's dimension theory is then used to produce examples of admissible families of $t$-worthy modules.

An example is given in $\S 6$ to show that the associated ideals, which are used to produce the extension of the tertiary theory, need not be prime ideals. Also it is shown that in certain special cases, our definition of associated ideal specializes to the usual definitions of associated prime ideal.

Presented to the Society, April 19, 1969; received by the editors December 3, 1968 and, in revised form, February 24, 1969.

( ${ }^{1}$ ) This research was partially supported by a National Science Foundation Summer Traineeship and is the author's doctoral dissertation at the University of Illinois. 
In [8] Riley gives an axiomatic characterization of the tertiary theory for an admissible family of finitely generated unitary modules over a left Noetherian ring with an identity. We prove in $\S 8$ that one of his axioms can be deduced from the others. Also an axiomatic characterization of the tertiary theory for an admissible family of $t$-worthy modules is given.

In $\$ \S 9-10$ the theory is applied to admissible families of $R$-modules where $R$ is a commutative ring. We prove that the decomposition theory constructed from the primary radical function on an admissible family of Noetherian modules is the classical primary theory. This result shows that it is possible to obtain the classical primary theory for a Noetherian module by using a technique similar to that of Bourbaki in [1].

We consider in $\S 10$ the radical function which is suggested by Bourbaki's definition of $\mathscr{P}$-primary in [1]. We call it the weakly primary radical function and we examine the decomposition theory that is obtained from it.

In $\$ 11$ we apply the theory to obtain generalizations of the Krull Intersection Theorem, the Lemma of Artin-Rees, and a result of Lesieur and Croisot.

1. Conventions and definitions. Unless explicitly mentioned to the contrary, throughout this paper, $R$ will denote an arbitrary associative ring. By this we mean that $R$ is not necessarily commutative, $R$ does not necessarily possess an identity, and $R$ does not necessarily possess any chain conditions (ascending or descending) on any family of left, right, or two-sided ideals. All $R$-modules will be assumed to be left $R$-modules. By a Noetherian $R$-module we mean an $R$-module with ascending chain condition on submodules. An $R$-module is said to be Artinian if it has descending chain condition on submodules. A left Noetherian ring is a ring that is Noetherian as a left module over itself. The term "ideal" will refer to a two-sided ideal unless it is adorned with the adjective "left". Also $\mathscr{M}$ will denote the category of all $R$-modules and $R$-homomorphisms.

Following the terminology of A. W. Goldie in [2], we define an $R$-module $M$ to be finite dimensional over $R$ if there does not exist an infinite chain of submodules of $M$ of the form $M_{1} \subset M_{1} \oplus M_{2} \subset M_{1} \oplus M_{2} \oplus M_{3} \subset \ldots$ where $M_{1}, M_{2}, \ldots$ are nonzero submodules of $M$. A submodule $U$ of $M$ is said to be uniform if $U \neq(0)$ and every pair of nonzero submodules of $U$ has nonzero intersection. A submodule $E$ of $M$ is called essential if $E$ has nonzero intersection with every nonzero submodule of $M$.

In [2] Goldie assumes that each of his $R$-modules, $M$, has the property that if $m$ is in $M$ and $R m=0$, then $m=0$. This assumption is not used in the proof of [2, Lemma 3.1]. Goldie's proof of [2, Lemma 3.2] can be slightly modified in order to give a proof which does not use this assumption. With this modification we have $3.1,3.2$, and 3.3 in [2] at our disposal without this assumption.

From [2, Theorem 3.3] we have that if $M$ is a finite dimensional $R$-module, then there exists a positive integer $n$ such that any direct sum of uniform submodules of 
$M$ having maximal length has $n$ terms and every direct sum of nonzero submodules of $M$ has at most $n$ terms. Furthermore a submodule of $M$ is essential if and only if it contains a direct sum of $n$ uniform submodules. Goldie calls this integer $n$, the dimension of $M$ over $R$ and denotes it by $\operatorname{dim} M$.

Let $M$ be an $R$-module. If $M$ is finite dimensional then $E$ is an essential submodule of $M$ if and only if $\operatorname{dim} E=\operatorname{dim} M$. Also $M$ is uniform if and only if $\operatorname{dim} M=1$. If $M=N_{1} \oplus N_{2}$ then $\operatorname{dim} M=\operatorname{dim} N_{1}+\operatorname{dim} N_{2}$. If $M$ is either a Noetherian or an Artinian $R$-module then $M$ is finite dimensional. There exist finite dimensional $R$-modules which are neither Noetherian nor Artinian. For example the rational numbers $Q$ are a 1-dimensional module over the rational integers $Z$; however, $Q$ is neither a Noetherian nor an Artinian $Z$-module.

The following definitions are taken from [4], [7], [8], and [9]. An ideal $\mathscr{P}$ in $R$ is called a prime ideal if for ideals $I, J$ in $R$, the product $I J \subseteq \mathscr{P}$ implies $I \subseteq \mathscr{P}$ or $J \subseteq \mathscr{P}$. If $R$ is commutative this definition is equivalent to the following: $\mathscr{P}$ is prime if whenever a product $r s$ of two elements of $R$ is in $\mathscr{P}$ then $r$ is in $\mathscr{P}$ or $s$ is in $\mathscr{P}$. If $\mathscr{A}$ is an ideal in $R$ then the primary radical of $\mathscr{A}$, denoted $p(\mathscr{A})$, is the intersection of all prime ideals in $R$ which contain $\mathscr{A}$. If $R$ is a commutative ring and $\mathscr{A}$ is an ideal in $R$, then from [9, p. 151], $p(\mathscr{A})=\left\{r \in R: r^{n} \in \mathscr{A}\right.$ for some positive integer $n$ \}. If $N^{\prime}$ and $N^{\prime \prime}$ are submodules of an $R$-module $M$, then the left residual quotient of $N^{\prime}$ and $N^{\prime \prime}$, denoted $\left(N^{\prime}: N^{\prime \prime}\right)$, is $\left\{r \in R: r N^{\prime \prime} \subseteq N^{\prime}\right\}$. If $M$ is an $R$-module, then the primary radical of $M$, denoted $p(M)$, is the primary radical of $(0: M)$. A submodule $N$ of $M$ is called primary if each $r$ in $R$, which annihilates a nonzero submodule of $M / N$, lies in $p(M / N)$. For a module over a commutative ring this definition of a primary submodule is equivalent to the one in $[9$, p. 252].

The tertiary radical of an $R$-module $M$, denoted $t(M)$, is defined to be $\{r \in R$ : there exists an essential submodule $E$ of $M$ with $r E=0\}$. The tertiary radical of an $R$-module is an ideal in $R$. A submodule $N$ of $M$ is called tertiary if each $r$ in $R$, which annihilates a nonzero submodule of $M / N$, lies in $t(M / N)$.

2. Radical functions. Let $R$ be a ring and let $C$ be a family of $R$-modules such that if $M$ is in $C$ and $0 \rightarrow M^{\prime} \rightarrow M \rightarrow M^{\prime \prime} \rightarrow 0$ is an exact sequence in $\mathscr{M}$, then $M^{\prime}$ and $M^{\prime \prime}$ are in $C$. A function, $r$, from $C$ to a family $B$ of ideals in $R$ is called a radical function on $C$ relative to $B$ if $M$ in $C$ and $0 \rightarrow M^{\prime} \rightarrow M$ exact in $\mathscr{M}$ implies that $r\left(M^{\prime}\right) \supseteq r(M)$.

Examples of radical functions on $\mathscr{M}$ are provided by $r(M)=(0: M)$ for $M$ in $\mathscr{M}$ and by the primary radical, $p$.

Lemma 2.1. Let $M$ be an $R$-module and let $0 \rightarrow M^{\prime} \rightarrow M$ be exact in $\mathscr{M}$. Then $t\left(M^{\prime}\right) \supseteq t(M)$. Moreover if $E$ is an essential submodule of $M$ then $t(E)=t(M)$.

Proof. It suffices to show that $t\left(M^{\prime}\right) \supseteq t(M)$ with $M^{\prime}$ any submodule of $M$. Let $r \in t(M)$. Then there exists an essential submodule $F$ of $M$ such that $r F=0$. If $M^{\prime}=(0)$ then the result follows immediately. If $M^{\prime} \neq(0)$ then $F \cap M^{\prime} \neq(0)$. Clearly 
$F \cap M^{\prime}$ is an essential submodule of $M^{\prime}$ and $r\left(F \cap M^{\prime}\right)=0$. Therefore $r \in t\left(M^{\prime}\right)$ and so $t\left(M^{\prime}\right) \supseteq t(M)$.

From the preceding paragraph, $t(E) \supseteq t(M)$. For the other inclusion let $r \in t(E)$. Then there exists an essential submodule $G$ of $E$ with $r G=0$. But $G$ is an essential submodule of $M$. Hence $r \in t(M)$.

From this lemma it follows that the tertiary radical, $t$, is also a radical function on $\mathscr{M}$.

Suppose that $r$ is a radical function on $C$. A module $S$ in $C$ is said to be $r$-stable if $S \neq(0)$ and for each nonzero submodule $N$ of $S, r(N)=r(S)$. Apparently every irreducible module $M$ in $C$ is $r$-stable. Lemma 2.1 shows that each uniform $R$ module is $t$-stable. Every nonzero submodule of an $r$-stable module is $r$-stable.

Let $M$ be in $C$. An ideal $\mathscr{A}$ in $R$ is called an associated ideal of $M$ with respect to $r$ if there exists an $r$-stable submodule $S$ of $M$ such that $\mathscr{A}=r(S)$. Let $A(M)$ denote the set of associated ideals of $M$. We notice that if $S$ is $r$-stable then $A(S)$ consists of the single ideal, $r(S)$. A most important property of $A(M)$ is that if $\mathscr{A}$ is in $A(M)$ then there exists an $r$-stable submodule $S$ of $M$ such that $A(S)=\{\mathscr{A}\}$.

The technique of using the following sequence of propositions in order to execute the proof of the main existence theorem in $\$ 4$ is patterned after that of Bourbaki in [1, pp. 131-146]. For the remainder of this section we will consider the same radical function $r$ on $C$.

Proposition 2.2. If $M$ is in $C$ and $0 \rightarrow M^{\prime} \rightarrow M \rightarrow M^{\prime \prime} \rightarrow 0$ is exact, then $A\left(M^{\prime}\right) \subseteq A(M) \subseteq A\left(M^{\prime}\right) \cup A\left(M^{\prime \prime}\right)$.

Proof. It is sufficient to consider $M^{\prime}$ as a submodule of $M$. Then the inclusion $A\left(M^{\prime}\right) \subseteq A(M)$ is evident.

Suppose that $\mathscr{A} \in A(M)$. Then there exists an $r$-stable submodule $S$ of $M$ such that $\mathscr{A}=r(S)$. If $M^{\prime} \cap S \neq(0)$ then $M^{\prime} \cap S$ is an $r$-stable submodule of $M^{\prime}$ and so $\mathscr{A} \in A\left(M^{\prime}\right)$. If $M^{\prime} \cap S=(0)$ then the submodule $M^{\prime}+S / M^{\prime}$ of $M / M^{\prime}$ is isomorphic to $S$. Therefore $M^{\prime}+S / M^{\prime}$ is $r$-stable and hence $\mathscr{A} \in A\left(M / M^{\prime}\right)=A\left(M^{\prime \prime}\right)$.

Proposition 2.3. If $M$ in $C$ is the (set theoretic) union of a family $\left\{M_{i}\right\}$ of submodules of $M$, then $A(M)=\bigcup A\left(M_{i}\right)$.

Proof. The inclusion $A(M) \supseteq \bigcup A\left(M_{i}\right)$ follows from 2.2. If $\mathscr{A} \in A(M)$ then there exists an $r$-stable submodule $S$ of $M$ such that $\mathscr{A}=r(S)$. Then $S \cap M_{i} \neq(0)$ for some $i$. Therefore $S \cap M_{i}$ is an $r$-stable submodule of $M_{i}$ and so $\mathscr{A} \in A\left(M_{i}\right)$.

Proposition 2.4. If $M$ in $C$ is the direct sum of a family $\left\{M_{i}: i \in I\right\}$, then

$$
A(M)=\bigcup A\left(M_{i}\right), \quad(i \in I) .
$$

Proof. By using 2.3 we can immediately reduce the proof to the case where $I$ is finite. By using induction on the cardinality of $I$ we can then reduce the proof to the case where $I$ has cardinality 2 . Hence suppose that $I=\{i, j\}, i \neq j$. Then $M / M_{i}$ 
is isomorphic to $M_{j}$ and we have that $A(M) \subseteq A\left(M_{i}\right) \cup A\left(M_{j}\right)$ by 2.2. The other inclusion $A\left(M_{i}\right) \cup A\left(M_{j}\right) \subseteq A(M)$ is evident.

Proposition 2.5. If $M$ is in $C$ and $E$ is an essential submodule of $M$, then $A(E)=A(M)$.

Proof. Proposition 2.2 shows that $A(E) \subseteq A(M)$. For the other inclusion let $\mathscr{A} \in A(M)$. Then there exists an $r$-stable submodule $S$ of $M$ such that $\mathscr{A}=r(S)$. Since $E$ is essential, $E \cap S$ is an $r$-stable submodule of $E$. Therefore $\mathscr{A} \in A(E)$.

Proposition 2.6. Let $M$ be in $C$. If $\mathscr{A}$ is in $A(M)$ then there exists a submodule $N$ of $M$ such that

(1) $A(M / N)=\{\mathscr{A}\}$,

(2) $A(N)=A(M)-\{\mathscr{A}\}$.

Also any submodule $N$ of $M$ which is maximal with respect to the property that $A(N) \subseteq A(M)-\{\mathscr{A}\}$ satisfies (1) and (2).

Proof. Let $\mathscr{F}$ be the family of submodules $W$ of $M$ such that $A(W) \subseteq A(M)-\{\mathscr{A}\}$. Partially order $\mathscr{F}$ by inclusion. Proposition 2.3 shows that $\mathscr{F}$ is inductive. Also $\mathscr{F} \neq \varnothing$ since $(0) \in \mathscr{F}$. By Zorn's Lemma there exists a maximal element $N$ in $\mathscr{F}$.

Since $\mathscr{A} \in A(M)$ and $A(N) \subseteq A(M)-\{\mathscr{A}\}$, it follows from 2.2 that $\mathscr{A} \in A(M / N)$. Let $\mathscr{B}$ be any element of $A(M / N)$. Then there exists an $r$-stable submodule $S / N$ of $M / N$ such that $\mathscr{B}=r(S / N)$. By Proposition 2.2, $A(S) \subseteq A(N) \cup A(S / N)$. Now $A(S / N)=\{\mathscr{B}\}$ and so $A(S) \subseteq A(N) \cup\{\mathscr{B}\}$. However $N$ is a maximal element of $\mathscr{F}$ and hence $N \varsubsetneqq S$ implies that $S \notin \mathscr{F}$. Therefore $\mathscr{A} \in A(S)$ but $\mathscr{A} \notin A(N)$. So $\mathscr{A}$ must be equal to $\mathscr{B}$. Consequently $A(M / N)=\{\mathscr{A}\}$. In view of 2.2 it now follows that $A(M)-\{\mathscr{A}\} \subseteq A(N)$. This completes the proof.

Suppose that $M$ is in $C$. For each $\mathscr{A} \in A(M)$, let $N(\mathscr{A})$ be a submodule of $M$ such that $A(M / N(\mathscr{A}))=\{\mathscr{A}\}$ and $A(N(\mathscr{A}))=A(M)-\{\mathscr{A}\}$. Consider

$$
\bigcap\{N(\mathscr{A}): \mathscr{A} \in A(M)\} \text {. }
$$

For each $\mathscr{A} \in A(M)$ we have that $A\left(\bigcap_{\mathscr{A} \in A(M)} N(\mathscr{A})\right) \subseteq A(N(\mathscr{A}))=A(M)-\{\mathscr{A}\}$. Therefore $A\left(\cap_{\mathscr{A} \in A(M)} N(\mathscr{A})\right)=\varnothing$. If $M$ has the property that for each submodule $N$ of $M, A(N)=\varnothing$ implies $N=(0)$, then we would have a decomposition of (0) in $M$.

LEMma 2.7. The following are equivalent for $M$ in $C$ :

(a) for each submodule $N$ of $M, A(N)=\varnothing$ if and only if $N=(0)$;

(b) each nonzero submodule of $M$ contains an r-stable submodule.

Proof. The equivalence follows immediately from the definitions.

An example of this type of situation is the following. Suppose that $M$ is a finite dimensional $R$-module. Goldie shows in [2, Lemma 3.1] that each nonzero submodule of $M$ contains a uniform submodule. Therefore each nonzero submodule of $M$ contains a $t$-stable submodule. 
The above argument proves the following:

THEOREM 2.8. If each nonzero submodule of $M$ in $C$ contains an r-stable submodule, then there exists a decomposition of (0) in $M$ of the form $(0)=\bigcap_{\mathscr{A} \in A(M)} N(\mathscr{A})$ where $A(M / N(\mathscr{A}))=\{\mathscr{A}\}$ for $\mathscr{A} \in A(M)$.

In this section we have started with a radical function $r$ on $C$ and we have obtained a decomposition of ( 0$)$ in $M$ for each $M$ in $C$ which has the property that each nonzero submodule contains an $r$-stable submodule. It should be noted that for such a module $M,\{N(\mathscr{A}): \mathscr{A} \in A(M)\}$ may be infinite. If this is the case, then this decomposition of (0) may not be irredundant, i.e., one such that no proper subset of $\{N(\mathscr{A}): \mathscr{A} \in A(M)\}$ has (0) for its intersection. See Example 2. In $\S 4$ we will see that $A(M)$ being finite is sufficient to guarantee the existence of an irredundant decomposition of $(0)$ in $M$.

3. Associated ideal functions. Let $R$ be a ring and let $C$ continue to denote the same family of $R$-modules as in $\S 2$. A function $\Gamma$ from $C$ to a family $D$ of sets of ideals of $R$ is called an associated ideal function on $C$ relative to $D$ if $M$ in $C$ and $0 \rightarrow M^{\prime} \rightarrow M$ exact imply that $\Gamma\left(M^{\prime}\right) \subseteq \Gamma(M)$. If $r$ is a radical function on $C$ and if $A(M)$ denotes the set of associated ideals of $M$ with respect to $r$ then Proposition 2.2 shows that $A$ is an associated ideal function on $M$.

Suppose that $\Gamma$ is an associated ideal function on $C$. A module $S$ in $C$ is said to be $\Gamma$-stable if $S \neq(0)$ and there exists an ideal $\mathscr{A}$ in $R$ such that for each nonzero submodule $N$ of $S, \Gamma(N)=\{\mathscr{A}\}$. We notice that each nonzero submodule of a $\Gamma$-stable submodule is $\Gamma$-stable. If $S$ is an $r$-stable submodule of $M$ then $S$ is $A$-stable.

From an associated ideal function $\Gamma$ on $C$, define $r_{\Gamma}(M)=\bigcap\{\mathscr{A}: \mathscr{A} \in \Gamma(M)\}$ for each $M$ in $C$.

Proposition 3.1. If $M$ is in $C$ and $0 \rightarrow M^{\prime} \rightarrow M$ is exact then $r_{\Gamma}\left(M^{\prime}\right) \supseteq r_{\Gamma}(M)$.

Proof. Since $\Gamma\left(M^{\prime}\right) \subseteq \Gamma(M)$ we have that

$$
\bigcap\left\{\mathscr{A}: \mathscr{A} \in \Gamma\left(M^{\prime}\right)\right\} \supseteq \bigcap\{\mathscr{A}: \mathscr{A} \in \Gamma(M)\} .
$$

Therefore $r_{\Gamma}\left(M^{\prime}\right) \supseteq r_{\Gamma}(M)$.

This proposition shows that from an associated ideal function $\Gamma$ on $C$, we can obtain a radical function $r_{\Gamma}$ on $C$. Now we will drop the $\Gamma$ on $r_{\Gamma}$ and use the notation $\Gamma \rightarrow r$ to indicate that $r$ is the radical function obtained in this way from $\Gamma$. Likewise we will use $r \rightarrow \Gamma$ to indicate that $\Gamma$ is the associated ideal function obtained from the radical function $r$ by the method of $\$ 2$.

Proposition 3.2. Let $r^{\prime}$ be a radical function on a family $C$ and let $r^{\prime} \rightarrow \Gamma^{\prime} \rightarrow r^{\prime \prime}$. For each $M$ in $C$,

$$
r^{\prime}(M) \subseteq r^{\prime \prime}(M)=\bigcap\left\{r^{\prime}(S): S \text { is an } r^{\prime} \text {-stable submodule of } M\right\} .
$$


Hence a necessary and sufficient condition for $r^{\prime}=r^{\prime \prime}$ is that for each $M$ in $C$, $\bigcap\left\{r^{\prime}(S): S\right.$ is an $r^{\prime}$-stable submodule of $\left.M\right\} \subseteq r^{\prime}(M)$.

Proof. Let $M \in C$. If $S$ is any submodule of $M$, then $r^{\prime}(M) \subseteq r^{\prime}(S)$. Therefore $r^{\prime}(M) \subseteq \bigcap\left\{r^{\prime}(S): S\right.$ is an $r^{\prime}$-stable submodule of $\left.M\right\}$. The rest of the proof is quite evident.

Proposition 3.3. Let $\Gamma$ be an associated ideal function on $C$ and let $\Gamma \rightarrow r^{\prime} \rightarrow$ $\Gamma^{\prime} \rightarrow r^{\prime \prime}$. If for each $M$ in $C$ and $\mathscr{A}$ in $\Gamma(M)$ there exists a $\Gamma$-stable submodule $S$ of $M$ such that $\Gamma(S)=\{\mathscr{A}\}$, then $r^{\prime}=r^{\prime \prime}$.

Proof. Let $M \in C$. If $\mathscr{A} \in \Gamma(M)$ then by hypothesis there exists a $\Gamma$-stable submodule $S$ of $M$ such that $\Gamma(S)=\{\mathscr{A}\}$. Since $\Gamma \rightarrow r^{\prime}$, we have that $S$ is also $r^{\prime}$-stable and $\mathscr{A}=r^{\prime}(S)$. Therefore $\Gamma(M) \subseteq\left\{r^{\prime}(S): S\right.$ is an $r^{\prime}$-stable submodule of $\left.M\right\}$. Thus

$\bigcap\left\{r^{\prime}(S): S\right.$ is an $r^{\prime}$-stable submodule of $\left.M\right\} \subseteq \bigcap\{\mathscr{A}: \mathscr{A} \in \Gamma(M)\}=r^{\prime}(M)$.

So 3.2 shows that $r^{\prime}=r^{\prime \prime}$.

Proposition 3.4. Let $r$ be a radical function on $C$. If $r \rightarrow \Gamma \rightarrow r^{\prime} \rightarrow \Gamma^{\prime} \rightarrow r^{\prime \prime}$, then $r^{\prime}=r^{\prime \prime}$.

Proof. Since $r \rightarrow \Gamma$, we have that for each $M \in C$ and $\mathscr{A} \in \Gamma(M)$ there exists an $r$-stable submodule $S$ of $M$ with $r(S)=\mathscr{A}$. Then $S$ is also $\Gamma$-stable and so the result follows from 3.3.

THEOREM 3.5. Let $\Gamma$ be an associated ideal function on $C$ and let $\Gamma \rightarrow r^{\prime} \rightarrow \Gamma^{\prime}$. Then $\Gamma=\Gamma^{\prime}$ if and only if the following conditions are satisfied:

(1) for each $M$ in $C, \Gamma(M)=\varnothing$ if and only if $M=(0)$, and

(2) for each $M$ in $C$ and $\mathscr{A}$ in $\Gamma(M)$ there exists a $\Gamma$-stable submodule $S$ of $M$ such that $\Gamma(S)=\{\mathscr{A}\}$.

Proof. Suppose that $\Gamma=\Gamma^{\prime}$ and $M \in C$. If $M=(0)$, then $\Gamma^{\prime}(M)=\varnothing$ because $r^{\prime}$-stable submodules of $M$ are nonzero by definition. Hence $\Gamma(M)=\varnothing$. If $M \neq(0)$ and $\Gamma(M)=\varnothing$, then $\Gamma(N)=\varnothing$ for each nonzero submodule $N$ of $M$. Therefore $r^{\prime}(N)=R$ for each nonzero submodule $N$ of $M$ since $r^{\prime}(N)$ is an empty intersection. Hence $M$ is $r^{\prime}$-stable and so $R \in \Gamma^{\prime}(M)$. This contradicts $\Gamma=\Gamma^{\prime}$. Therefore (1).

In order to show (2) let $\mathscr{A} \in \Gamma^{\prime}(M)$. Then there exists an $r^{\prime}$-stable submodule $S$ of $M$ such that $\mathscr{A}=r^{\prime}(S)$. Since $S$ is $r^{\prime}$-stable it is $\Gamma^{\prime}$-stable with $\Gamma^{\prime}(S)=\{\mathscr{A}\}$. So (2) follows because $\Gamma=\Gamma^{\prime}$.

Suppose that (1) and (2) hold. Let $M \in C$ and $\mathscr{A} \in \Gamma(M)$. From (2) there exists a $\Gamma$-stable submodule $S$ of $M$ such that $\Gamma(S)=\{\mathscr{A}\}$. Since $\Gamma \rightarrow r^{\prime}, S$ is also $r^{\prime}$-stable and $r^{\prime}(S)=\mathscr{A}$. Therefore $\mathscr{A} \in \Gamma^{\prime}(M)$.

Now let $\mathscr{A} \in \Gamma^{\prime}(M)$. Then there exists an $r^{\prime}$-stable submodule $N$ of $M$ such that $\mathscr{A}=r^{\prime}(N)$. Since $N \neq(0), \Gamma(N) \neq \varnothing$ by (1). If $\mathscr{B} \in \Gamma(N)$, then (2) shows the existence of a $\Gamma$-stable submodule $S$ of $N$ with $\Gamma(S)=\{\mathscr{B}\}$. Moreover $r^{\prime}(S)=\mathscr{B}$. Therefore $\mathscr{A}=r^{\prime}(N)=r^{\prime}(S)=\mathscr{B}$ and so $\mathscr{A} \in \Gamma(M)$. 
Proposition 3.6. Let $r$ be a radical function on $C$ and let $r \rightarrow \Gamma \rightarrow r^{\prime} \rightarrow \Gamma^{\prime}$. Then $\Gamma=\Gamma^{\prime}$ if and only if each nonzero $M$ in $C$ contains an $r$-stable submodule.

Proof. If $\Gamma=\Gamma^{\prime}$ and $M$ is a nonzero module in $C$, then 3.5 yields that $\Gamma(M) \neq \varnothing$. Since $r \rightarrow \Gamma$, there exists an $r$-stable submodule of $M$.

Suppose that each nonzero $M \in C$ contains an $r$-stable submodule. Then $\Gamma(M)$ $=\varnothing$ if and only if $M=(0)$. Furthermore for each $M \in C$ and $\mathscr{A} \in \Gamma(M)$, there is an $r$-stable submodule $S$ of $M$ with $\mathscr{A}=r(S)$. Since $S$ is $r$-stable, it is also $\Gamma$-stable and $\Gamma(S)=\{\mathscr{A}\}$. Therefore $\Gamma=\Gamma^{\prime}$ by 3.5 .

4. Decomposition theories. Let $R$ be a ring. A family $C$ of $R$-modules is called an admissible family for a decomposition theory if the following conditions are satisfied:

(1) if $M$ is in $C$ and $0 \rightarrow M^{\prime} \rightarrow M \rightarrow M^{\prime \prime} \rightarrow 0$ is an exact sequence in $\mathscr{M}$, then $M^{\prime}$ and $M^{\prime \prime}$ are in $C$; and

(2) if $M^{\prime}$ and $M^{\prime \prime}$ are in $C$, then $M^{\prime} \oplus M^{\prime \prime}$ is in $C$.

The family of all Noetherian $R$-modules is an example of an admissible family for a decomposition theory. We now prepare to define a most important collection of admissible families.

Let $r$ be a radical function on an admissible family $C$ of $R$-modules and let $A$ be the associated ideal function on $C$ that is obtained from $r$. An $R$-module $M$ is said to be worthy of a decomposition theory with respect to $r$ or just $r$-worthy if each factor module $M^{\prime \prime}$ of $M$ satisfies the following conditions:

(a) each nonzero submodule of $M^{\prime \prime}$ contains an $r$-stable submodule, and

(b) $A\left(M^{\prime \prime}\right)$ is finite.

If a section of an $R$-module $M$ is defined to be a factor module of a submodule of $M$, then (a) can be equivalently stated as follows: each nonzero section of $M$ contains an $r$-stable submodule.

Proposition 4.1. Suppose that $r$ is a radical function on an admissible family $C$ of $R$-modules, $M$ is in $C$, and $0 \rightarrow M^{\prime} \rightarrow M \rightarrow M^{\prime \prime} \rightarrow 0$ is an exact sequence in $\mathscr{M}$. Then $M$ is $r$-worthy if and only if both $M^{\prime}$ and $M^{\prime \prime}$ are r-worthy.

Proof. It is sufficient to consider $M^{\prime}$ as a submodule of $M$ and $M^{\prime \prime}$ as the factor module $M / M^{\prime}$.

Assume that $M$ is $r$-worthy. Since each factor module of $M^{\prime}$ is a section of $M$, it follows that $M^{\prime}$ is $r$-worthy. Also each factor module of $M^{\prime \prime}$ is isomorphic to a factor module of $M$. Hence $M^{\prime \prime}$ is $r$-worthy.

Assume that both $M^{\prime}$ and $M^{\prime \prime}$ are $r$-worthy. Suppose that $S$ is a nonzero section of $M$. Then $S$ has the form $N / N^{\prime}$ where $N^{\prime}, N$ are submodules of $M$. If $N^{\prime} \cap M^{\prime}$ $\varsubsetneqq N \cap M^{\prime}$ then the section $N \cap M^{\prime} / N^{\prime} \cap M^{\prime}$ of $M^{\prime}$ is isomorphic to a nonzero submodule of $N / N^{\prime}$. So $S$ contains an $r$-stable submodule. If $N^{\prime} \cap M^{\prime}=N \cap M^{\prime}$ then $\left(N+M^{\prime} / M^{\prime}\right) /\left(N^{\prime}+M^{\prime} / M^{\prime}\right) \cong N / N^{\prime}$. Consequently the worthiness of $M^{\prime \prime}$ gives an $r$-stable submodule of $S$. Therefore $M$ satisfies (a). 
In order to show that $M$ satisfies (b), let $M / P$ be a factor module of $M$. Consider the following existing isomorphisms:

$$
\frac{M^{\prime}+P}{P} \cong \frac{M^{\prime}}{M^{\prime} \cap P} \text { and } \quad \frac{M}{M^{\prime}+P} \cong \frac{M / M^{\prime}}{M^{\prime}+P / M^{\prime}}
$$

Proposition 2.2 yields that

$$
A\left(\frac{M}{P}\right) \subseteq A\left(\frac{M^{\prime}+P}{P}\right) \cup A\left(\frac{M}{M^{\prime}+P}\right)=A\left(\frac{M^{\prime}}{M^{\prime} \cap P}\right) \cup A\left(\frac{M / M^{\prime}}{M^{\prime}+P / M^{\prime}}\right) .
$$

Since both $M^{\prime}$ and $M^{\prime \prime}$ satisfy (b), the union of the two sets on the right is finite. Therefore $A(M / P)$ is finite. It follows that $M$ is $r$-worthy.

This proposition shows that the subfamily of all $r$-worthy $R$-modules in $C$ is an admissible family for a decomposition theory. It results from this that any $r$-worthy $R$-module can be imbedded in an admissible family of $r$-worthy $R$-modules.

Let $C$ be an admissible family for a decomposition theory. An associated ideal function $\Gamma$ on $C$ relative to $D$ is called a decomposition function if for each $M$ in $C$ and $0 \rightarrow M^{\prime} \rightarrow M \rightarrow M^{\prime \prime} \rightarrow 0$ exact, $\Gamma(M) \subseteq \Gamma\left(M^{\prime}\right) \cup \Gamma\left(M^{\prime \prime}\right)$.

If $r$ is a radical function on an admissible family $C$, then Proposition 2.2 shows that the associated ideal function that is obtained from $r$ is a decomposition function.

Proposition 4.2. Let $\Gamma$ be a decomposition function on an admissible family $C$. If $M$ is in $C$ and $0 \rightarrow M^{\prime} \rightarrow M \rightarrow M^{\prime \prime} \rightarrow 0$ is exact, then

$$
r_{\Gamma}\left(M^{\prime}\right) \supseteq r_{\Gamma}(M) \supseteq r_{\Gamma}\left(M^{\prime}\right) \cap r_{\Gamma}\left(M^{\prime \prime}\right) .
$$

Proof. The inclusion $r_{\Gamma}\left(M^{\prime}\right) \supseteq r_{\Gamma}(M)$ follows from 3.1. Since $\Gamma(M) \subseteq \Gamma\left(M^{\prime}\right)$ $\cup \Gamma\left(M^{\prime \prime}\right)$ we have that $r_{\Gamma}\left(M^{\prime}\right) \cap r_{\Gamma}\left(M^{\prime \prime}\right) \subseteq r_{\Gamma}(M)$.

Proposition 4.3. Let $\Gamma$ be a decomposition function on an admissible family $C$. Suppose that $\left\{N_{i}\right\}$ is a finite set of modules in $C$. Then

(i) $\Gamma\left(\Sigma^{\oplus} N_{i}\right)=\bigcup \Gamma\left(N_{i}\right)$,

(ii) $r_{\Gamma}\left(\Sigma^{\oplus} N_{i}\right)=\bigcap r_{\Gamma}\left(N_{i}\right)$.

Proof. We have that (i) follows immediately by induction from the definition of a decomposition function. And (ii) follows from (i) by definition of $r_{\Gamma}$.

Proposition 4.4. Let $\Gamma$ be a decomposition function on an admissible family $C$. Suppose that $M$ is in $C$ and $\left\{N_{i}\right\}$ is a finite set of submodules of $M$. If $(0)=\bigcap N_{i}$, then

(i) $\Gamma(M) \subseteq \bigcup \Gamma\left(M / N_{i}\right)$,

(ii) $r_{\Gamma}(M) \supseteq \bigcap r_{\Gamma}\left(M / N_{i}\right)$.

Proof. The canonical mapping $M \rightarrow \Sigma^{\oplus} M / N_{i}$ is injective. Therefore

$$
\Gamma(M) \subseteq \Gamma\left(\sum^{\oplus} M / N_{i}\right)=\bigcup \Gamma\left(M / N_{i}\right) .
$$

Likewise (ii) follows.

Let $\Gamma$ be an associated ideal function on an admissible family $C$. Suppose $M / N$ 
is in $C$. We say that $N$ is a $\Gamma$-submodule of $M$ if $M / N$ is $\Gamma$-stable. An element $M$ in $C$ is said to be $\Gamma$-finite if $\Gamma(M)$ is a finite set. A finite set $\left\{N_{i}: i \in I\right\}$ of submodules of $M$ in $C$ is a $\Gamma$-decomposition of $N$ in $M$ if the following conditions are satisfied:

(1) $\bigcap_{i \in I} N_{i}=N$ and for no $i \in I$ is $\bigcap_{j \neq i} N_{j} \subseteq N_{i}$;

(2) the $N_{i}, i \in I$ are $\Gamma$-submodules of $M$; and

(3) $\Gamma\left(M / N_{i}\right) \neq \Gamma\left(M / N_{j}\right)$ for $i \neq j$.

Notice that if $I$ is empty then the empty intersection, $\bigcap\left\{N_{i}: i \in I\right\}=M$ and so $M / N$ is zero.

Proposition 4.5. Suppose that $\Gamma$ is a decomposition function on an admissible family $C, M$ is in $C$, and $\left\{N_{i}\right\}$ is a $\Gamma$-decomposition of $(0)$ in $M$ with $\Gamma\left(M / N_{i}\right)=\left\{\mathscr{A}_{i}\right\}$. Then:

(a) $\Gamma(M)=\bigcup_{i}\left\{\mathscr{A}_{i}\right\}$,

(b) $\Gamma\left(N_{i}\right)=\bigcup_{j \neq i}\left\{\mathscr{A}_{j}\right\}$, and

(c) $\bigcap_{j \neq i} N_{j}$ is $\Gamma$-stable with $\Gamma\left(\bigcap_{j \neq i} N_{j}\right)=\left\{\mathscr{A}_{i}\right\}$.

Proof. Since $\left\{N_{i}\right\}$ is a $\Gamma$-decomposition of $(0)$ in $M, \bigcap_{j \neq i} N_{j} \neq(0)$. Moreover $\bigcap_{j \neq i} N_{j}$ is isomorphic to a nonzero submodule of $M / N_{i}$. So $\bigcap_{j \neq i} N_{j}$ is $\Gamma$-stable because $M / N_{i}$ is $\Gamma$-stable. Hence $\Gamma\left(\bigcap_{j \neq i} N_{j}\right)=\left\{\mathscr{A}_{i}\right\}$. Therefore (c).

Proposition 4.4 shows that $\Gamma(M) \subseteq \bigcup_{i} \Gamma\left(M / N_{i}\right)=\bigcup_{i}\left\{\mathscr{A}_{i}\right\}$. For each $i,\left\{\mathscr{A}_{i}\right\}=$ $\Gamma\left(\bigcap_{j \neq i} N_{j}\right) \subseteq \Gamma(M)$. Therefore (a).

Since $N_{i} \cap\left(\bigcap_{j \neq i} N_{j}\right)=(0), N_{i}$ is isomorphic to a submodule of $M / \bigcap_{j \neq i} N_{j}$. So $\Gamma\left(N_{i}\right) \subseteq \Gamma\left(M / \bigcap_{j \neq i} N_{j}\right)$. Proposition 4.4 shows that $\Gamma\left(M / \bigcap_{j \neq i} N_{j}\right)=\bigcup_{j \neq i}\left\{\mathscr{A}_{j}\right\}$. Hence $\Gamma\left(N_{i}\right) \subseteq \bigcup_{j \neq i}\left\{\mathscr{A}_{j}\right\}$. Because $\Gamma$ is a decomposition function, $\Gamma(M) \subseteq \Gamma\left(N_{i}\right)$ $\cup \Gamma\left(M / N_{i}\right)=\Gamma\left(N_{i}\right) \cup\left\{\mathscr{A}_{i}\right\}$. Therefore $\bigcup_{j \neq i}\left\{\mathscr{A}_{j}\right\} \subseteq \Gamma\left(N_{i}\right)$. Hence (b).

Proposition 4.6. Suppose that $\Gamma$ is a decomposition function on an admissible family $C, M$ is in $C$, and (0) has a $\Gamma$-decomposition in $M$. Then each nonzero submodule of $M$ contains a $\Gamma$-stable submodule.

Proof. Let $\left\{N_{i}: i \in I\right\}$ be a $\Gamma$-decomposition of $(0)$ in $M$. We use induction on the cardinality of $I$. If the cardinality of $I$ is 0 then $M=(0)$ and the proposition is vacuous. If the cardinality of $I$ is 1 , then $N_{1}=(0)$ and $M$ is $\Gamma$-stable. Therefore each nonzero submodule of $M$ is $\Gamma$-stable.

Assume that the conclusion holds for any $M$ and $I$ for which the cardinality of $I$ is less than $n$. Let $I$ have cardinality $n, n>1$, and let $N$ be a nonzero submodule of $M$. If $N \cap\left(\bigcap_{j \neq 1} N_{j}\right) \neq(0)$, then $N \cap\left(\bigcap_{j \neq 1} N_{j}\right)$ is a $\Gamma$-stable submodule of $N$ because $\bigcap_{j \neq 1} N_{j}$ is $\Gamma$-stable by 4.5(c). If $N \cap\left(\bigcap_{j \neq 1} N_{j}\right)=(0)$, then $N$ is isomorphic to a nonzero submodule of $M / \bigcap_{j \neq 1} N_{j}$. The set $\left\{N_{i} / \bigcap_{j \neq 1} N_{j}: i=2, \ldots, n\right\}$ is a $\Gamma$-decomposition of (0) in $M / \bigcap_{j \neq 1} N_{j}$. Therefore by induction, $N$ contains a $\Gamma$-stable submodule.

Proposition 4.7. Suppose that $\Gamma$ is a decomposition function on an admissible family $C, M$ is in $C$, and (0) has a $\Gamma$-decomposition in $M$. Then:

(1) $\Gamma(M)=\varnothing$ if and only if $M=(0)$, 
(2) $M$ is $\Gamma$-finite, and

(3) for each $\mathscr{A}$ in $\Gamma(M)$, there exists a $\Gamma$-stable submodule $S$ of $M$ such that $\Gamma(S)=\{\mathscr{A}\}$.

Proof. If $M \neq(0)$ then 4.6 shows that $M$ contains a $\Gamma$-stable submodule $S$. Then $\Gamma(S) \subseteq \Gamma(M)$ is nonempty. Hence $\Gamma(M) \neq \varnothing$. Suppose that $\Gamma(M) \neq \varnothing$. Then 4.5(c) shows that $M$ contains a $\Gamma$-stable submodule which is nonzero by definition. So $M \neq(0)$. Hence (1).

Now (2) follows from 4.5(a) and (3) follows from 4.5(c).

Proposition 4.8. Suppose that $\Gamma$ is a decomposition function on an admissible family $C, M$ is in $C$, and $\left\{N_{i}\right\}$ is a $\Gamma$-decomposition of $(0)$ in $M$ with $\Gamma\left(M / N_{i}\right)=\left\{\mathscr{A}_{i}\right\}$. Let $M\left(\mathscr{A}_{i}\right)=\bigcap_{j \neq i} N_{j}$. Then:

(1) if $N$ is a submodule of $M$ and $\mathscr{A}_{i}$ is in $\Gamma(N)$, then $N \cap M\left(\mathscr{A}_{i}\right) \neq(0)$, and

(2) $\sum_{i}^{\oplus} M\left(\mathscr{A}_{i}\right)$ is an essential submodule of $M$.

Proof. If $N \cap M\left(\mathscr{A}_{i}\right)=(0)$, then $N$ is isomorphic to a submodule of $M / M\left(\mathscr{A}_{i}\right)$. So $\Gamma(N) \subseteq \Gamma\left(M / M\left(\mathscr{A}_{i}\right)\right)$. Proposition 4.4 shows that $\Gamma\left(M / M\left(\mathscr{A}_{i}\right)\right) \subseteq \bigcup_{j \neq i}\left\{\mathscr{A}_{j}\right\}$. Therefore $\Gamma(N) \subseteq \bigcup_{j \neq i}\left\{\mathscr{A}_{j}\right\}$. This contradicts $\mathscr{A}_{i} \in \Gamma(N)$. Hence $N \cap M\left(\mathscr{A}_{i}\right) \neq(0)$.

The $\sum_{i} M\left(\mathscr{A}_{i}\right)$ in (2) is direct since $\bigcap_{i} N_{i}=(0)$. Let $N$ be any nonzero submodule of $M$. Proposition 4.6 shows that $N$ contains a $\Gamma$-stable submodule $S$. Now $\Gamma(S)=\left\{\mathscr{A}_{i}\right\}$ for some $i$ because $\Gamma(S) \subseteq \Gamma(M)$. Then (1) implies that $N \cap M\left(\mathscr{A}_{i}\right) \neq(0)$ because $\mathscr{A}_{i} \in \Gamma(N)$. Therefore $N \cap\left(\sum_{i}^{\oplus} M\left(\mathscr{A}_{i}\right)\right) \neq(0)$. Hence (2).

THEOREM 4.9. Suppose that $r$ is a radical function on an admissible family $C, A$ is the associated ideal function on $C$ that is obtained from $r$, and $M$ is in $C$. Then (0) has an A-decomposition in $M$ if and only if

(a) each nonzero submodule of $M$ contains an r-stable submodule, and

(b) $M$ is A-finite.

Proof. Suppose that (0) has an $A$-decomposition in $M$ and $N$ is a nonzero submodule of $M$. Proposition 4.6 shows that $N$ contains an $A$-stable submodule $S$. Since $A(S) \neq \varnothing$ and $r \rightarrow A$, we have that $N$ contains an $r$-stable submodule. Statement (b) follows from 4.7.

Suppose that (a) and (b) hold. For each $\mathscr{A} \in A(M)$ choose a submodule $N(\mathscr{A})$ of $M$ which is maximal with respect to $A(N(\mathscr{A})) \subseteq A(M)-\{\mathscr{A}\}$. Proposition 2.6 shows that $A(M / N(\mathscr{A}))=\{\mathscr{A}\}$ and $A(N(\mathscr{A}))=A(M)-\{\mathscr{A}\}$. By the argument preceding Lemma 2.7, we have that $A\left(\bigcap_{\mathscr{A} \in A(M)} N(\mathscr{A})\right)=\varnothing$. Lemma 2.7 produces that $\bigcap_{\mathscr{A} \in A(M)} N(\mathscr{A})=(0)$.

We claim that $M / N(\mathscr{A})$ is $A$-stable for each $\mathscr{A} \in A(M)$. Suppose that $S / N(\mathscr{A})$ is a nonzero submodule of $M / N(\mathscr{A})$. Then $S \supsetneqq N(\mathscr{A})$ and $A(S) \subseteq A(N(\mathscr{A})) \cup$ $A(S / N(\mathscr{A})$ ). Since $N(\mathscr{A})$ is maximal with respect to $A(N(\mathscr{A})) \subseteq A(M)-\{\mathscr{A}\}$, we have that $\mathscr{A} \in A(S)$ but $\mathscr{A} \notin A(N(\mathscr{A}))$. Hence $\mathscr{A} \in A(S / N(\mathscr{A}))$. Therefore

$$
A(S / N(\mathscr{A}))=\{\mathscr{A}\}
$$

and $M / N(\mathscr{A})$ is $A$-stable. 
All that remains to be shown is that for no $\mathscr{B} \in A(M)$ is $\bigcap \mathscr{A} \neq \mathscr{B} N(\mathscr{A}) \subseteq N(\mathscr{B})$. If for some $\mathscr{B} \in A(M), \bigcap_{\mathscr{A} \neq \mathscr{B}} N(\mathscr{A}) \subseteq N(\mathscr{B})$; then $\bigcap_{\mathscr{A} \neq \mathscr{B}} N(\mathscr{A})=(0)$. Since $A(M)$ is finite, Proposition 4.4 shows that $A(M) \subseteq \bigcup_{\mathscr{A} \neq \mathscr{B}} A(M / N(\mathscr{A}))=A(M)-\{\mathscr{B}\}$. This is a contradiction. Hence the theorem is proved.

Suppose that $\Gamma$ is a decomposition function on an admissible family $C$. We say that $\Gamma$ is a decomposition theory on $C$ if for each $M$ in $C$, (0) has a $\Gamma$-decomposition in $M$.

THEOREM 4.10. Let $r$ be a radical function on an admissible family $C$ and let $A$ be the associated ideal function on $C$ that is obtained from $r$. A necessary and sufficient condition that $A$ be a decomposition theory on $C$ is that each module in $C$ be $r$-worthy.

Proof. Assume that $A$ is a decomposition theory on $C$. In order to show that each module in $C$ is $r$-worthy, it suffices to show that each $M$ in $C$ satisfies (a) and (b) of Theorem 4.9. Indeed these follow from 4.9.

The sufficiency of the condition also follows from 4.9.

THEOREM 4.11. Let $\Gamma$ be a decomposition theory on an admissible family $C$. Then it is possible to obtain $\Gamma$ from the radical function $r_{\Gamma}$ by using the technique of $\S 2$.

Proof. Consider $\Gamma \rightarrow r_{\Gamma} \rightarrow A$. From 4.7 and 3.5 we have that $\Gamma=A$. Therefore $r_{\Gamma} \rightarrow \Gamma$.

In view of this result it appears that the technique of constructing a decomposition theory from a radical function is the natural way to construct decomposition theories.

THEOREM 4.12. Let $\Gamma$ be a decomposition function on an admissible family $C$. Then $\Gamma$ is a decomposition theory on $C$ if and only if

(1) for each $M$ in $C, \Gamma(M)=\varnothing$ if and only if $M=(0)$;

(2) each $M$ in $C$ is $\Gamma$-finite; and

(3) for each $M$ in $C$ and $\mathscr{A}$ in $\Gamma(M)$, there exists a $\Gamma$-stable submodule $S$ of $M$ such that $\Gamma(S)=\{\mathscr{A}\}$.

Proof. Proposition 4.7 yields the necessity.

In order to show sufficiency consider $\Gamma \rightarrow r \rightarrow A$. Theorem 3.5 shows that $\Gamma=A$. We claim that $C$ is an admissible family of $r$-worthy modules. In order to show this, it suffices to show that each nonzero module in $C$ contains an $r$-stable submodule. Let $M$ be a nonzero module in $C$. Then $\Gamma(M) \neq \varnothing$. Suppose that $\mathscr{A} \in \Gamma(M)$. Then by (3) there exists a $\Gamma$-stable submodule $S$ of $M$ with $\Gamma(S)=\{\mathscr{A}\}$. Also $S$ is $r$-stable and hence $C$ is an admissible family of $r$-worthy modules. The sufficiency now follows from 4.10; in that, $A$ is a decomposition theory on $C$.

Proposition 4.13. Let $\Gamma$ be a decomposition theory on an admissible family $C$ and let $M$ be in $C$. Then for each essential submodule $E$ of $M$ :

(1) $\Gamma(E)=\Gamma(M)$ and

(2) $r_{\Gamma}(E)=r_{\Gamma}(M)$. 
Proof. From 4.11 we have that $r_{\Gamma} \rightarrow \Gamma$. Therefore (1) follows from Lemma 2.5. Conclusion (2) follows immediately from (1).

Corollary 4.14. Let $\Gamma$ be a decomposition theory on an admissible family $C$. If $U$ in $C$ is uniform, then

(i) $U$ is $\Gamma$-stable, and

(ii) $U$ is $r_{\Gamma}$-stable.

Proof. The proof is evident.

Proposition 4.15. Let $\Gamma$ be a decomposition theory on an admissible family $C$ and let $M$ be a nonzero module in $C$. Then (0) is a $\Gamma$-submodule of $M$ if and only if $M$ is $r_{\Gamma}$-stable.

Proof. If (0) is a $\Gamma$-submodule of $M$, then $M$ is $\Gamma$-stable and hence $M$ is $r_{\Gamma}$-stable. From 4.11 we have that $r_{\Gamma} \rightarrow \Gamma$. Therefore if $M$ is $r_{\Gamma}$-stable, then $M$ is $\Gamma$-stable. Wherefore (0) is a $\Gamma$-submodule of $M$.

Let $\Gamma, \Omega$ be two decomposition theories on an admissible family $C$. Then $\Gamma$ is said to be smaller than $\Omega$ on $C$ if for each $M$ in $C, r_{\Gamma}(M) \subseteq r_{\Omega}(M)$. In terms of the ideals associated to $M$ in $C$ by $\Gamma$ and $\Omega$, " $\Gamma$ smaller than $\Omega$ on $C$ " says that each ideal in $\Gamma(M)$ is contained in one or more ideals of $\Omega(M)$. In order to show this let $\mathscr{A}$ be in $\Gamma(M)$. Then there exists a $\Gamma$-stable submodule $S$ of $M$ such that $\Gamma(S)=$ $\{\mathscr{A}\}$. Thus $\mathscr{A}=r_{\Gamma}(S) \subseteq r_{\Omega}(S)=\bigcap_{\mathscr{B} \in \Omega(S)} \mathscr{B}$. Note that $\Omega(S)$ is nonempty since $S \neq(0)$ and $\Omega$ is decomposition theory.

A decomposition theory $\Gamma$ on an admissible family $C$ is said to be normal on $C$ if it has the following two properties:

(a) if $M$ is in $C$ and $\mathscr{A}$ is in $\Gamma(M)$, then $\mathscr{A} \supseteq(0: M)$; and

(b) $\Gamma$ is the smallest decomposition theory on $C$ which satisfies (a), i.e., if $\Omega$ is any decomposition theory on $C$ which satisfies (a), then $\Gamma$ is smaller than $\Omega$ on $C$. We notice that (a) is equivalent to the following: for each $M$ in $C, r_{\Gamma}(M) \supseteq(0: M)$.

Suppose that $\Gamma$ is a decomposition theory on an admissible family $C$ of $R$ modules. If $I$ is an ideal in $R$, then the set of all $M$ in $C$ that are annihilated by $I$ is an admissible family $D$ of $R / I$-modules. Suppose that for each $M$ in $D$ and $\mathscr{A}$ in $\Gamma(M), \mathscr{A} \supseteq I$. Then the function $\Gamma_{R / I}$ defined on $M$ in $D$ by $\Gamma_{R / I}(M)=\{\mathscr{A} / I: \mathscr{A}$ is in $\Gamma(M)\}$ is an associated ideal function on $D$. Therefore property (a) in the definition of normality simply guarantees that for each ideal $I$ in $R, \Gamma_{R / I}$ is defined on $D$.

By $\Gamma$ being a normal decomposition theory on $C$, we are roughly saying that $\Gamma$ is the decomposition theory on $C$ which assigns to each $M$ in $C$ the "smallest" ideals which contain $(0: M)$.

THEOREM 4.16. Let $\Gamma, \Omega$ be two normal decomposition theories on an admissible family $C$. Then $\Gamma=\Omega$ on $C$.

Proof. Since $\Gamma, \Omega$ are normal on $C$, we have that for each $M \in C, r_{\Gamma}(M)=r_{\Omega}(M)$. Theorem 4.11 shows that $r_{\Gamma} \rightarrow \Gamma$ and $r_{\Omega} \rightarrow \Omega$. Therefore $\Gamma=\Omega$ because $r_{\Gamma}=r_{\Omega}$. 
COROllaRy 4.17. If $\Gamma$ is a normal decomposition theory on $C$ then $\Gamma$ is the unique normal decomposition theory on $C$.

5. Existence of the tertiary theory. In $\S 2$ we saw that the tertiary radical, $t$, is a radical function on $\mathscr{M}$. Now we will examine the associated ideal function that is obtained from $t$. Suppose that $M$ is an $R$-module. An ideal $\mathscr{A}$ in $R$ is an associated ideal of $M$ with respect to $t$ if there exists a $t$-stable submodule $S$ of $M$ such that $\mathscr{A}=t(S)$. Denote the set of associated ideals of $M$ by $T(M)$. We have that $T$ is an associated ideal function on $\mathscr{M}$. Theorem 4.10 shows that $T$ is a decomposition theory on an admissible family $C$ if and only if each module in $C$ is $t$-worthy.

A finite set $\left\{N_{i}: i \in I\right\}$ of submodules of an $R$-module $M$ is a teritary decomposition of $N$ in $M$ if the following conditions are satisfied:

(1) $\bigcap_{i \in I} N_{i}=N$ and for no $i \in I$ is $\bigcap_{j \neq i} N_{j} \subseteq N_{i}$;

(2) the $N_{i}, i \in I$, are tertiary submodules of $M$; and

(3) $t\left(M / N_{i}\right) \neq t\left(M / N_{j}\right)$ for $i \neq j$.

We will proceed to show that a decomposition is a tertiary decomposition if and only if it is a $T$-decomposition.

Lemma 5.1. Let $N$ be an $R$-module such that each nonzero submodule of $N$ contains a t-stable submodule. Let $\Delta$ denote the set of all $t$-stable submodules of $N$. Then every sum of the form, $\sum_{s \in \Delta} N_{S}$ where $N_{S}$ is a nonzero submodule of $S$, is an essential submodule of $N$.

Proof. Let $W$ be a nonzero submodule of $N$. Then $W$ contains a $t$-stable submodule $V$. Since $V \in \Delta$, we have that $W \cap \sum_{S \in \Delta} N_{S} \supseteq N_{V} \neq(0)$. Therefore $\sum_{s \in \Delta} N_{S}$ is essential.

Proposition 5.2. Let $M$ be an R-module such that each nonzero submodule of $M$ contains a t-stable submodule. Then for each nonzero submodule $N$ of $M, t(N)=r_{T}(N)$ where $t \rightarrow T \rightarrow r_{T}$.

Proof. Let $\Delta$ denote the set of all $t$-stable submodules of $N$. It follows from 3.2 that it is sufficient to show that $\bigcap_{s \in \Delta} t(S) \subseteq t(N)$. Suppose that $r \in \bigcap_{s \in \Delta} t(S)$. Then for each $S \in \Delta$ there exists a nonzero submodule $N_{S}$ of $S$ such that $r N_{S}=0$. Lemma 5.1 shows that $\sum_{S \in \Delta} N_{S}$ is an essential submodule of $N$. Since $r\left(\sum_{S \in \Delta} N_{S}\right)=0$, we have that $r \in t(N)$.

THEOREM 5.3. Let $M$ be an R-module. Then the following are equivalent:

(a) (0) is a $T$-submodule of $M$ with $T(M)=\{\mathscr{A}\}$;

(b) (0) is a tertiary submodule of $M$ with $t(M)=\mathscr{A}$;

(c) $M$ is $t$-stable with $t(M)=\mathscr{A}$.

Proof. Assume that (a) holds. Then each nonzero submodule $W$ of $M$ contains a $t$-stable submodule since $\mathscr{A} \in T(W)$. Therefore Proposition 5.2 shows that for each nonzero submodule $N$ of $M, t(N)=r_{T}(N)=\mathscr{A}$. In particular $t(M)=\mathscr{A}$. Let 
$r \in R$ annihilate a nonzero submodule $S$ of $M$. Then $r \in t(S)=\mathscr{A}$. Hence $r \in t(M)$ and so by definition ( 0 ) is tertiary. Whence (a) implies (b).

Assume that (b) holds. Suppose that $N$ is a nonzero submodule of $M$. If $r \in t(N)$, then $r$ annihilates a nonzero submodule of $M$. Thus $r \in t(M)$ since (0) is tertiary. So $t(N) \subseteq t(M)$. The other inclusion follows from 2.1. Therefore $t(N)=t(M)$ and $M$ is $t$-stable. Hence (b) implies (c).

That (c) implies (a) is evident.

This theorem shows that a decomposition is a tertiary decomposition if and only if it is a $T$-decomposition. Therefore, if $T$ is a decomposition theory on an admissible family $C$, then we call $T$ the tertiary decomposition theory on $C$. We now have the following important theorem as a corollary of Theorem 4.10.

THEOREM 5.4. Let $R$ be an arbitrary ring and $C$ an admissible family of $R$-modules. Then a necessary and sufficient condition that the tertiary decomposition theory exists on $C$ is that each module in $C$ be $t$-worthy.

At this point it might be rather difficult for the reader to visualize an admissible family of $t$-worthy $R$-modules. Therefore we will proceed to investigate a collection of rather nice admissible families of $t$-worthy $R$-modules.

Proposition 5.5. Suppose that $M$ is an R-module which is $n$ dimensional over $R$. Then the cardinality of $T(M)$ is at most $n$.

Proof. From [2, Theorem 3.3] we have that there exists an essential submodule $E$ of $M$ of the form $E=U_{1} \oplus U_{2} \oplus \cdots \oplus U_{n}$, where $U_{i}, 1 \leqq i \leqq n$, are uniform. Proposition 2.5 shows that $T(M)=T(E)$. And $T(E)=\bigcup_{i=1}^{n} T\left(U_{i}\right)$ by 2.4. Since for each $i, 1 \leqq i \leqq n, U_{i}$ is uniform, the set $T\left(U_{i}\right)$ consists of a single ideal. Therefore the cardinality of $T(M)$ is at most $n$.

As we remarked in $\$ 2$, each nonzero submodule of a finite dimensional $R$ module contains a $t$-stable submodule. In view of this and 5.5 we have the following proposition:

Proposition 5.6. If each factor module of an $R$-module $M$ is finite dimensional over $R$, then $M$ is $t$-worthy.

From this proposition we see that an admissible family of finite dimensional $R$-modules is an admissible family of $t$-worthy $R$-modules. If $M$ is a Noetherian $R$-module, then each factor module of $M$ is Noetherian. Hence each factor module of $M$ is finite dimensional over $R$. Likewise if $M$ is Artinian, then each factor module of $M$ is finite dimensional over $R$. Consequently if $M$ is either Noetherian or Artinian, it is $t$-worthy. This proves the following corollary of 5.4.

COROLlary 5.7. Suppose that $R$ is an arbitrary ring and that $C$ is either an admissible family of Artinian R-modules or an admissible family of Noetherian $R$-modules. Then for each $M$ in $C$, there exists a tertiary decomposition of (0) in $M$. 
Lesieur and Croisot have proved the above corollary in [7] with the following additional and unnecessary assumption on each $M$ in $C$ :

Assumption 1. For each submodule $N^{\prime}$ of $M, R$ has the ascending chain condition on ideals of the form $\left(N^{\prime}: N^{\prime \prime}\right), N^{\prime \prime}$ a submodule of $M$.

6. Associated ideals. One question, above all others, led to the writing of this paper. It is the following. Let $R$ be an arbitrary ring and let $M$ be a Noetherian $R$-module. Is it possible to associate prime ideals in $R$ to the submodules of $M$ in some way, in order to obtain some kind of a decomposition of the submodules of $M$ ? If we are willing to put the ascending chain condition on a certain family of ideals of $R$ as set forth in Assumption 1, then the answer is "yes". We would associate a prime ideal $\mathscr{P}$ in $R$ to $M$ if and only if there exists a nonzero submodule $N$ of $M$ such that for each nonzero submodule $W$ of $N, \mathscr{P}=(0: W)$. Denote this set of associated prime ideals of $M$ by Ass $(M)$. Then, as Riley has shown in [8], we would obtain the tertiary decomposition theory. If we are not willing to put this assumption on $R$, then what ? With the possible sacrifice of the primeness of the associated ideals of $M$, we have shown in $\S 5$ that we can obtain a decomposition theory for a Noetherian $R$-module. To our delight we still get the tertiary decomposition theory. To our amazement we still get the tertiary decomposition theory even for a much more general module than a Noetherian module. The following example shows however that we did in fact sacrifice the primeness of the associated ideals.

EXAMPLE 1. Let $V$ be a countably infinite dimensional vector space over a field $F$. Let $\left\{v_{1}, v_{2}, \ldots\right\}$ be a basis for $V$. For each positive integer $i$, consider the subspace $V_{i}$ of $V$ which is spanned by $\left\{v_{i}, v_{i+1}, v_{i+2}, \ldots\right\}$. Let $\mathscr{A}_{1}$ be the ring which consists of those transformations, $\tau$, on $V$ such that

(1) for each positive integer $i, \tau V_{i} \subseteq V_{i}$, and

(2) for which there exists a positive integer $j$ such that $\tau V_{j}=0$.

Define the linear transformation $s$ on $V$ by $s v_{i}=v_{i+1}$ for $i$ odd and $s v_{i}=0$ for $i$ even. Note that $s^{2}=0$. Let $R$ be the ring generated over $\mathscr{A}_{1}$ by $s$. Then $\mathscr{A}_{1}$ is an ideal in $R$.

It can be shown that the proper submodules of the $R$-module, $V$, are precisely the $V_{i}, i=2,3, \ldots$ Hence $V$ is Noetherian, non-Artinian, and uniform as an $R$-module.

The tertiary radical of $V$ is $\mathscr{A}_{1}$. Since $V$ is uniform, $T(V)=\left\{\mathscr{A}_{1}\right\}$. However $\mathscr{A}_{1}$ is not prime since $R \nsubseteq \mathscr{A}_{1}$ but $R^{2} \subseteq \mathscr{A}_{1}$.

Suppose that $M$ is an $R$-module which satisfies Assumption 1. We will show that with this assumption the associated ideals in $T(M)$ become prime and our definition of associated ideal is equivalent to the usual definition [8, p. 186] of associated prime ideal; that being, a prime ideal in Ass $(M)$.

Proposition 6.1. Let $M$ be an R-module which satisfies Assumption 1. Then $\mathscr{A}$ is in $T(M)$ if and only if $\mathscr{A}$ is in Ass $(M)$. 
Proof. Suppose that $\mathscr{A} \in T(M)$. Then there exists a $t$-stable submodule $U$ of $M$ with $\mathscr{A}=t(U)$. Consider $\mathscr{F}=\{(0: S): S$ is a nonzero submodule of $U\}$. By Assumption 1 there exists a maximal element $(0: N)$ in $\mathscr{F}$. Since $(0: N)$ is maximal, $(0: W)=(0: N)$ for each nonzero submodule $W$ of $N$. Therefore $\mathscr{A}=t(N)=(0: N)$ and so $\mathscr{A}=(0: W)$ for each nonzero submodule $W$ of $N$.

In order to show that $\mathscr{A}$ is prime, suppose that $I J \subseteq \mathscr{A}, I, J$ ideals in $R$. If $J \nsubseteq \mathscr{A}$ then $J N \neq(0)$ and hence $I \subseteq(0: J N)=\mathscr{A}$. Wherefore $\mathscr{A}$ is prime and so $\mathscr{A} \in$ Ass $(M)$.

Suppose that $\mathscr{P} \in$ Ass $(M)$. Then there exists a nonzero submodule $N$ of $M$ such that for each nonzero submodule $W$ of $N, \mathscr{P}=(0: W)$. Thence $N$ is $t$-stable and $\mathscr{P}=t(N)$. Accordingly $\mathscr{P} \in T(M)$.

Proposition 6.2. Let $R$ be a commutative ring and $M$ an $R$-module. If $\mathscr{A}$ is in $T(M)$, then $\mathscr{A}$ is prime.

Proof. Since $\mathscr{A} \in T(M)$, there exists a $t$-stable submodule $U$ of $M$ with $\mathscr{A}=t(U)$. Suppose that $r, s \in R$ with $r s \in t(U)$. Then there is a nonzero submodule $V$ of $U$ such that $(r s) V=0$. If $s \notin t(U)$ then $s V \neq 0$. However $r(s V)=0$ and so $r \in t(s V)=\mathscr{A}$. Therefore $\mathscr{A}$ is prime.

By using the associated ideal function, $T$, we will now give an example of a module $M$ where the decomposition of $(0)$ in $M$ given by $(0)=\bigcap \mathscr{A} \in T(M) N(\mathscr{A})$ is not irredundant.

EXAmple 2. Let $F, V, V_{i}$ for $i=1,2, \ldots$, and $\mathscr{A}_{1}$ be as in Example 1. Set $R=\mathscr{A}_{1} \oplus F I$ where $I$ is the identity transformation on $V$. It can be shown that the proper submodules of the $R$-module, $V$, are precisely the $V_{i}, i=2,3, \ldots$

Let $M$ be the $R$-module, $M=V_{1} \oplus V / V_{2} \oplus V / V_{3} \oplus \cdots$. We claim that each nonzero submodule of $M$ contains a $t$-stable submodule. Suppose that $N$ is a nonzero submodule of $M$. Then there exists a positive integer $k$ and $n_{1} \in V_{1}$, $n_{2} \in V / V_{2}, \ldots$ such that $n=\left(n_{1}, n_{2}, \ldots, n_{k}, 0, \ldots\right)$ is in $N$. Hence $R n \subseteq R n_{1} \oplus R n_{2}$ $\oplus \cdots \oplus R n_{k}$. For each $i, V / V_{i}$ is a 1-dimensional $R$-module in the sense of Goldie. So $R n_{1} \oplus R n_{2} \oplus \cdots \oplus R n_{k}$ is a $k$-dimensional $R$-module in the sense of Goldie. Thence it follows from [2, Lemma 3.1] that $R n$ contains a uniform submodule. Therefore $N$ contains a uniform submodule which is necessarily $t$-stable.

Proposition 2.4 shows that $T(M)=T\left(V_{1}\right) \cup T\left(V / V_{2}\right) \cup \ldots$. The $V / V_{i}$ for $i=2,3, \ldots$ are uniform so that $T\left(V / V_{i}\right)=\left\{t\left(V / V_{i}\right)\right\}=\left\{\left(V_{i}: V_{i-1}\right)\right\}$. Set $\mathscr{A}_{i}=\left(V_{i}: V_{i-1}\right)$ for $i=2,3, \ldots$ Since $T\left(V_{1}\right)=\left\{\mathscr{A}_{1}\right\}$, we have that $T(M)=\bigcup_{i=1}^{\infty}\left\{\mathscr{A}_{i}\right\}$. The $\mathscr{A}_{i}$, $i=1,2, \ldots$ are distinct maximal ideals in $R$.

For each $\mathscr{A}_{i}$ in $T(M)$, one choice for the $N\left(\mathscr{A}_{i}\right)$ in Proposition 2.6 is $N\left(\mathscr{A}_{1}\right)=$ $\sum_{j=2}^{\infty} V / V_{j}$ and $N\left(\mathscr{A}_{i}\right)=V_{1} \oplus \sum_{j \neq i}^{\oplus} V / V_{j}$ for $i \geqq 2$. From these $N\left(\mathscr{A}_{i}\right), i=1,2, \ldots$ we will construct a new choice which is intersection redundant.

If an $R$-module $P$ is the direct sum of two submodules $N$ and $S$, then for each $R$-homomorphism $\phi: N \rightarrow S$, the monomorphism $f: n \rightarrow n+\phi(n)$ takes $N$ onto another complement, $f(N)$, of $S$ in $P$. For each $i=2,3, \ldots$ define $\phi_{i}: N\left(\mathscr{A}_{i}\right) \rightarrow V / V_{i}$ 
by $\phi_{i}:\left(x_{j}: j \neq i\right) \rightarrow x_{1}+V_{i}$. In this manner we obtain for $i=2,3, \ldots$ a complement $f_{i} N\left(\mathscr{A}_{i}\right)$ of $V / V_{i}$ in $M$ where $f_{i}: N\left(\mathscr{A}_{i}\right) \rightarrow M$ is given by

$$
f_{i}:\left(x_{j}: j \neq i\right) \rightarrow\left(x_{j}: x_{i}=x_{1}+V_{i}\right) .
$$

Set $f_{1} N\left(\mathscr{A}_{1}\right)=\sum_{j=2}^{\infty} \underset{\oplus}{\oplus} V / V_{j}$.

For each $i=1,2, \ldots$ we have that $T\left(M / f_{i} N\left(\mathscr{A}_{i}\right)\right)=\left\{\mathscr{A}_{i}\right\}$ and $T\left(f_{i} N\left(\mathscr{A}_{i}\right)\right)=T(M)$ $-\left\{\mathscr{A}_{i}\right\}$. Therefore $(0)=\bigcap_{i=1}^{\infty} f_{i} N\left(\mathscr{A}_{i}\right)$.

We claim that $\bigcap_{i=2}^{\infty} f_{i} N\left(\mathscr{A}_{i}\right)=(0)$. Suppose that $x=\left(x_{j}: j=1,2, \ldots\right) \in M$ is in $\bigcap_{i=2}^{\infty} f_{i} N\left(\mathscr{A}_{i}\right)$. An element $y=\left(y_{j}: j=1,2, \ldots\right) \in M$ is in $f_{i} N\left(\mathscr{A}_{i}\right)$ if and only if $y_{i}=y_{1}+V_{i}$. Hence $x_{j}=x_{1}+V_{j}$ for $j=2,3, \ldots$ Assume that $x_{1} \neq 0$. Since $V_{i} \supset V_{i+1}$ for $i=1,2, \ldots$ and $\bigcap_{i=1}^{\infty} V_{i}=0$, there exists a positive integer $k$ such that $x_{1} \notin V_{i}$ for $i>k$. Therefore we have reached a contradiction because $x_{1}+V_{j}$ a nonzero element of $V / V_{j}$ for $j>k$ contradicts the fact that $x$ in the direct sum must have only finitely many $x_{j}$ nonzero. Consequently $x_{1}=0$ and so $x=0$ since $x_{j}=x_{1}+V_{j}$ for $j=2,3, \ldots$.

\section{Uniqueness of the tertiary theory.}

Proposition 7.1. On an admissible family $C$ of $t$-worthy $R$-modules, the tertiary decomposition theory, $T$, is normal.

Proof. Certainly if $M \in C$ and $\mathscr{A} \in T(M)$, then $\mathscr{A} \supseteq(0: M)$.

Let $\Omega$ be a decomposition theory on $C$ such that if $M \in C$ and $\mathscr{A} \in \Omega(M)$ then $\mathscr{A} \supseteq(0: M)$. Suppose that $M \in C$ and $s \in t(M)$. Then there exists an essential submodule $E$ of $M$ such that $s E=0$. Moreover $s \in(0: E) \subseteq r_{\Omega}(E)$. Proposition 4.13 shows that $r_{\Omega}(E)=r_{\Omega}(M)$. Therefore $t(M) \subseteq r_{\Omega}(M)$. From 5.2 we have that $r_{T}(M)=$ $t(M)$ for each $M \in C$. Thus $T$ is smaller than $\Omega$ on $C$ and so $T$ is normal on $C$.

From 4.17 we get the following theorem:

THEOREM 7.2. On an admissible family $C$ of $t$-worthy $R$-modules, the tertiary theory, $T$, is the unique normal decomposition theory.

Goldie [2, Chapter 7, p. 12] raised the question as to whether the tertiary theory is the only one that "provides all the salient features of an ideal theory" in the sense of the classical theory in commutative Noetherian rings. Riley has shown in [8] that the answer to this question is "yes", in the sense that, if $R$ is a left Noetherian ring with an identity and $C$ is an admissible family of finitely generated unitary $R$-modules, then with certain reasonable assumptions (normality in the sense of Riley), the tertiary theory is the only one that does provide all the salient features of an ideal theory. Theorem 7.2 answers Goldie's question in the affirmative even in this more general setting. In particular it shows that the tertiary theory is the only natural generalization to Noetherian modules over an arbitrary ring of the classical primary theory for Noetherian modules over a commutative ring.

A decomposition theory $\Gamma$ on an admissible family $C$ is said to be normal in the sense of Riley if for each $M$ in $C$, the elements of $\Gamma(M)$ (1) are annihilating ideals 
for $M$, i.e., if $\mathscr{A}$ is in $\Gamma(M)$ then there is a nonzero submodule $N$ of $M$ with $\mathscr{A} N=0$; and (2) contain $(0: M)$. While Riley's definition of a decomposition theory differs slightly from ours, these two properties are the essential features of his definition of normality. We will show that if $\Gamma$ is normal in the sense of Riley then it is normal; however, there exist decomposition theories which are normal in our sense but not in Riley's sense.

Proposition 7.3. Let $\Gamma$ be a decomposition theory on an admissible family $C$. If $\Gamma$ is normal in the sense of Riley then $\Gamma$ is normal.

Proof. Suppose that $\Omega$ is a decomposition theory on $C$ such that if $M \in C$ and $\mathscr{B} \in \Omega(M)$ then $\mathscr{B} \supseteq(0: M)$. In order to show that $r_{\Gamma}(M) \subseteq r_{\Omega}(M)$ for each $M \in C$, it is sufficient to show that for each $M \in C$ and $\mathscr{B} \in \Omega(M)$, there is an $\mathscr{A} \in \Gamma(M)$ such that $\mathscr{A} \subseteq \mathscr{B}$.

Assume that $M \in C$ and $\mathscr{B} \in \Omega(M)$. Then there exists an $\Omega$-stable submodule $S$ of $M$ with $\Omega(S)=\{\mathscr{B}\}$. Since $S \neq(0)$, there is an $\mathscr{A}$ in $\Gamma(S)$. Because $\Gamma$ is normal in the sense of Riley there exists a nonzero submodule $N$ of $S$ with $\mathscr{A} \subseteq(0: N)$. We have that $\Omega(N)=\{\mathscr{B}\}$ and so $(0: N) \subseteq \mathscr{B}$. Hence $\mathscr{A} \subseteq \mathscr{B}$. Thence $r_{\Gamma}(M) \subseteq r_{\Omega}(M)$ for each $M$ in $C$ and so $\Gamma$ is smaller than $\Omega$ on $C$. Whence $\Gamma$ is normal.

Example 3. Consider the $R$-module $V$ of Example 1. Each factor module of $V$ is 1-dimensional over $R$. Proposition 5.6 shows therefore that $V$ is $t$-worthy. So $V$ is contained in an admissible family $C$ of $t$-worthy $R$-modules. The tertiary decomposition theory, $T$, exists and is normal on $C$ by 5.4 and 7.1. However $T$ is not normal in the sense of Riley on $C$ because $\mathscr{A}_{1}$ is in $T(V)$, but $\mathscr{A}_{1}$ is not an annihilating ideal for $V$. Thus these two concepts of normality do not in general coincide.

Proposition 7.4. Suppose that $C$ is an admissible family of t-worthy $R$-modules such that each module in $C$ satisfies Assumption 1. If a decomposition theory $\Gamma$ is normal on $C$ then it is normal in the sense of Riley.

Proof. From Theorem 7.2, $\Gamma=T$. If $M \in C$ and $\mathscr{A} \in \Gamma(M)$, then 6.1 shows that $\mathscr{A} \in$ Ass $(M)$. Therefore $\mathscr{A}$ is an annihilating ideal for $M$ and $\mathscr{A} \supseteq(0: M)$. Whence $\Gamma$ is normal in the sense of Riley on $C$.

8. Axiomatic tertiary theory. Let $R$ be a left Noetherian ring with an identity and $C$ an admissible family of finitely generated unitary $R$-modules. In [8] Riley has shown that five axioms for an associated ideal function, $A$, on $C$ provide an axiomatic characterization of the tertiary decomposition theory, $T$, on $C$, as it is given in this special case. See $\S 6$. These five axioms are as follows:

(i) For $M \in C, A(M)=\varnothing$ if and only if $M=(0)$.

(ii) If $M \in C$ is the (set theoretic) union of a family $\left\{M_{i}\right\}$ of submodules, then $A(M)=\bigcup_{i} A\left(M_{i}\right)$.

(iii) If $M \in C$ and $0 \rightarrow M^{\prime} \rightarrow M \rightarrow M^{\prime \prime} \rightarrow 0$ is exact, then

$$
A(M) \subseteq A\left(M^{\prime}\right) \cup A\left(M^{\prime \prime}\right) .
$$


(iv) If $M \neq(0)$ is in $C$, then there exists, for each $\mathscr{P} \in A(M)$, a submodule $M^{\prime}$ of $M$ such that $A\left(M^{\prime}\right)=\{\mathscr{P}\}$.

(v) For $M \in C$, the elements of $A(M)$ are annihilating ideals for $M$ and each contains the annihilator of $M$.

We will show that axiom (ii) is not independent of the other four axioms and therefore it can be deleted.

Suppose that $M$ is the union of the family $\left\{M_{i}\right\}$ of submodules. The inclusion $\bigcup_{i} A\left(M_{i}\right) \subseteq A(M)$ follows immediately from the fact that $A$ is an associated ideal function. In order to show the other inclusion let $\mathscr{P} \in A(M)$. By axiom (iv) there exists a submodule $M^{\prime}$ of $M$ with $A\left(M^{\prime}\right)=\{\mathscr{P}\}$. Axiom (i) shows that $M^{\prime} \neq(0)$. Since $M$ is the set theoretic union of the $\left\{M_{i}\right\}$, there is an $M_{i}$ such that $M_{i} \cap M^{\prime} \neq(0)$. Hence $A\left(M_{i} \cap M^{\prime}\right) \neq \varnothing$. Because $A\left(M_{i} \cap M^{\prime}\right) \subseteq A\left(M^{\prime}\right)$ we now have that $A\left(M_{i} \cap M^{\prime}\right)=\{\mathscr{P}\}$. Thus $\mathscr{P} \in A\left(M_{i}\right)$ since $A\left(M_{i} \cap M^{\prime}\right) \subseteq A\left(M_{i}\right)$. Therefore $A(M) \subseteq$ $\bigcup_{i} A\left(M_{i}\right)$ and so axiom (ii) results. By relying on the results of Riley in [8], we have the following theorem:

THEOREM 8.1. The four axioms, (i), (iii), (iv), and (v) for an associated ideal function, $A$, on an admissible family $C$ of finitely generated unitary modules over a left Noetherian ring $R$ with an identity, provide an axiomatic characterization of the tertiary decomposition theory, $T$, on $C$.

We now state the following theorem in order to summarize the axiomatic characterization of the tertiary theory which we have obtained in this paper.

THEOREM 8.2. Let $R$ be an arbitrary ring. The following five axioms for an associated ideal function, $A$, on an admissible family $C$ of $t$-worthy $R$-modules, provide an axiomatic characterization of the tertiary decomposition theory, $T$, on $C$ :

(a) For $M \in C, A(M)=\varnothing$ if and only if $M=(0)$.

(b) If $M \in C$ and $0 \rightarrow M^{\prime} \rightarrow M \rightarrow M^{\prime \prime} \rightarrow 0$ is exact, then

$$
A(M) \subseteq A\left(M^{\prime}\right) \cup A\left(M^{\prime \prime}\right) .
$$

(c) If $M \neq(0)$ is in $C$, then there exists, for each $\mathscr{A} \in A(M)$, a submodule $M^{\prime}$ of $M$ such that $A\left(M^{\prime}\right)=\{\mathscr{A}\}$.

(d) Each $M \in C$ is A-finite.

(e) $A$ is normal on $C$.

REMARK. Theorem 4.12 and the first four axioms force $A$ to be a decomposition theory on $C$. Therefore it makes sense to speak of $A$ as being normal in axiom (e).

Proof of 8.2. As remarked above, $A$ is a decomposition theory on $C$. Since axiom (e) makes $A$ normal on $C$, we have that $A=T$ by Theorem 7.2. Conversely, the fact that the tertiary theory, $T$, satisfies these five axioms on an admissible family of $t$-worthy $R$-modules follows from the way that we constructed $T$ from $t$ and from 7.1 .

We finish this section by showing that Theorem 8.1 can be obtained as a special 
case of 8.2. Suppose that $R$ is a left Noetherian ring with an identity and that $C$ is an admissible family of finitely generated unitary $R$-modules. Axiom (d) can be obtained from axioms (a), (b), and (c) by using a proof similar to that of $[8$, Corollary 2.4]. Then Theorem 4.12 shows that if $A$ satisfies axioms (a), (b), and (c) on $C$, it is a decomposition theory. Thus Propositions 7.3 and 7.4 show that together with axioms (a), (b), and (c); axiom (e) is equivalent to axiom (v) on $C$. Therefore on $C$ axioms (a)-(e) are equivalent to axioms (i), (iii), (iv), and (v).

9. The primary theory. Throughout this paragraph we will assume that $R$ is a commutative ring. Suppose that $C$ is an admissible family of $R$-modules. The primary radical, $p$, is a radical function on $C$. As in $\S 2$ an ideal $\mathscr{A}$ in $R$ is an associated ideal of $M$ in $C$ with respect to $p$ if there exists a $p$-stable submodule $S$ of $M$ such that $\mathscr{A}=p(S)$. Denote the set of associated ideals of $M$ by $P(M)$. We propose that the associated ideals of $M$ are prime ideals in $R$.

Proposition 9.1. If $M$ is in $C$ and $\mathscr{A}$ is in $P(M)$, then $\mathscr{A}$ is prime.

Proof. Since $\mathscr{A} \in P(M)$, there is a $p$-stable submodule $S$ of $M$ with $\mathscr{A}=p(S)$. Suppose that $x y \in \mathscr{A}$ for $x, y \in R$. Then there is an integer $n$ such that $(x y)^{n} S=0$. If $y \notin \mathscr{A}$ then $y^{n} S \neq 0$. Thus $x^{n}\left(y^{n} S\right)=0$ and so $x \in p\left(y^{n} S\right)$. However $S$ is $p$-stable so that $p\left(y^{n} S\right)=\mathscr{A}$ and hence $x \in \mathscr{A}$. Therefore $\mathscr{A}$ is prime.

Suppose that $C$ is an admissible family of $p$-worthy $R$-modules. Theorem 4.10 shows that the associated ideal function, $P$, is a decomposition theory on $C$. It would appear that this decomposition theory is the primary theory and that we have extended the primary theory to a larger family of modules than the family of Noetherian $R$-modules. Unfortunately $P$ is not the usual primary theory [9, p. 252] because a $P$-submodule does not necessarily need to be a primary submodule.

EXAMPLE 4. Let $V$ be a countably infinite dimensional vector space over a field $F$. Let $\left\{v_{1}, v_{2}, \ldots\right\}$ be a basis for $V$. For each positive integer $i$, consider the subspace $V_{i}$ of $V$ which is spanned by $\left\{v_{1}, v_{2}, \ldots, v_{i}\right\}$. Define the linear transformation $T$ on $V$ by $T v_{1}=0$ and $T v_{i}=v_{i-1}$ for $i \geqq 2$. Let $F[T]$ be the polynomial ring generated over $F$ by the transformation $T$. Then $F[T]$ is a commutative Noetherian ring with an identity.

Consider $V$ as an $F[T]$-module. It can be shown that the proper submodules of $V$ are precisely the $V_{i}, i=1,2, \ldots$ Hence $V$ is Artinian, non-Noetherian, and uniform as an $F[T]$-module.

Let $\mathscr{P}$ denote

$\left\{f(T) \in F[T]: f(T)=f_{1} T+f_{2} T^{2}+\cdots+f_{n} T^{n}\right.$ where $n$ is a positive integer and $\left.f_{i} \in F\right\}$.

Then $\mathscr{P}=p\left(V_{i}\right)$ for $i=1,2, \ldots$ and so $(0)$ is a $P$-submodule of $V$.

Now (0) is not a primary submodule of $V$ because $T \in F[T]$ annihilates the nonzero submodule $V_{1}$; however, $T$ is not in $p(V)$ since for each integer $i$,

$$
T^{i} \notin(0: V)=(0) \text {. }
$$


We will proceed to show that for a Noetherian module $M, P$-submodules are primary. While the following lemma appears in the literature in certain special cases, I have not found it stated in this generality.

Lemma 9.2. Let $R$ be a commutative ring and $M$ a Noetherian $R$-module. Then $p(M)=t(M)$.

Proof. Let $r \in R$ be in $p(M)$. Then there is a positive integer $n$ such that $r^{n} \in(0: M)$. Consider the submodule $(0: r)=\{m \in M: r m=0\}$ of $M$. Suppose that $N$ is a nonzero submodule of $M$. If $r N=0$ then $(0: r) \cap N \neq(0)$. If $r N \neq 0$, then there exists an $x \in N$ such that $r^{i} x \neq 0$ and $r^{i+1} x=0$ for some $i, 1 \leqq i<n$. Consequently

$$
(0: r) \cap N \neq(0)
$$

and so $(0: r)$ is an essential submodule of $M$. Therefore $r \in t(M)$.

Let $r \in R$ be in $t(M)$. For each $n, n=1,2, \ldots$ consider the submodule

$$
\left(0: r^{n}\right)=\left\{m \in M: r^{n} m=0\right\}
$$

of $M$. Since $M$ is Noetherian, we have for large $n,\left(0: r^{n}\right)=\left(0: r^{n+1}\right)=\cdots$. Now $r \in t(M)$ implies that there exists an essential submodule $E$ of $M$ such that $r E=0$. If $r^{n} M \neq 0$ then $E \cap r^{n} M \neq(0)$. Hence there exists an $m \in M$ such that $r^{n} m \neq 0$ but $r\left(r^{n} m\right)=0$. However this contradicts $\left(0: r^{n}\right)=\left(0: r^{n+1}\right)$. Therefore $r^{n} M=0$ and so $r \in p(M)$.

Proposition 9.3. Let $R$ be a commutative ring and $M$ a Noetherian $R$-module. Then the following are equivalent:

(a) (0) is a P-submodule of $M$;

(b) (0) is a tertiary submodule of $M$;

(c) (0) is a primary submodule of $M$.

Proof. Since $p(M)=t(M)$, it is immediate that (b) and (c) are equivalent.

Moreover ( 0$)$ is a $P$-submodule of $M$ if and only if (0) is a $T$-submodule of $M$. Therefore the equivalence of (a) and (b) follows from Theorem 5.3.

Suppose that $M$ is a Noetherian module over a commutative ring $R$. An interesting question is this. Can one appropriately define associated prime ideals for $M$ and then obtain the classical primary decomposition theory for $M$ by using a technique similar to that of Bourbaki in [1, pp. 131-146]? It is now clear that this is possible. We would define an associated prime ideal for $M$ as a prime ideal $\mathscr{P}$ for which there exists a $p$-stable submodule $S$ of $M$ such that $\mathscr{P}=p(S)$. The above proposition shows that a submodule of $M$ is a primary submodule if and only if it is a $P$-submodule. Therefore in this special case Theorem 4.10 gives the classical primary decomposition theory. We notice that an equivalent, but perhaps conceptually easier way to define, an associated prime ideal for $M$, would be as a prime ideal which is the tertiary (or primary) radical of a uniform submodule of $M$.

In order to obtain the classical primary theory for a finitely generated unitary module, $M$, over a Noetherian ring, $R$, with an identity; Bourbaki in [1, p. 131] 
defines an associated prime ideal as a prime ideal $\mathscr{P}$ in $R$ for which there exists a nonzero element $x$ in $M$ such that $\mathscr{P}=(0: x)$. The following proposition shows that with the additional assumption that $R$ is Noetherian, our definition of associated prime ideal for a Noetherian module, reduces to Bourbaki's definition.

Proposition 9.4. Suppose that $R$ is a commutative Noetherian ring with an identity and $M$ is a finitely generated unitary $R$-module. A prime ideal $\mathscr{P}$ is in $P(M)$ if and only if there exists a nonzero $x$ in $M$ such that $\mathscr{P}=(0: x)$.

Proof. Lemma 9.2 shows that a prime ideal $\mathscr{P} \in P(M)$ if and only if $\mathscr{P} \in T(M)$. Since $R$ is Noetherian and $M$ is $t$-worthy, Proposition 6.1 shows that $\mathscr{P} \in T(M)$ if and only if $\mathscr{P} \in \operatorname{Ass}(M)$. Riley in [8, p. 186] proves that $\mathscr{P} \in$ Ass $(M)$ if and only if there exists a nonzero $x$ in $M$ such that $\mathscr{P}=(0: x)$.

10. The weakly primary theory. Throughout this section we will assume that $R$ is a commutative ring. In [1, p. 139] Bourbaki introduces the concept of a $\mathscr{P}$ primary submodule of an $R$-module $M$. We make the following definition: A submodule $N$ of $M$ is called weakly primary if when $r \in R$ annihilates a nonzero submodule of $M / N$, then for each $\bar{x} \in M / N$ there exists a positive integer $n$ such that $r^{n} \bar{x}=\overline{0}$. Proposition 1 in [1, p. 139] shows that if $R$ is a Noetherian ring with an identity, then the concepts of $\mathscr{P}$-primary and weakly primary are equivalent.

The definition of a weakly primary submodule immediately suggests the notion of a weakly primary radical. If $M$ is an $R$-module, then the weakly primary radical of $M$, denoted $w(M)$, is defined to be

$$
\left\{r \in R \text { : for each } x \in M \text { there exists a positive integer } n \text { with } r^{n} x=0\right\} .
$$

On an admissible family $C$ of $R$-modules, the weakly primary radical is a radical function. As in $\S 2$ an ideal $\mathscr{A}$ in $R$ is an associated ideal of $M$ in $C$ with respect to $w$ if there exists a $w$-stable submodule $S$ of $M$ such that $\mathscr{A}=w(S)$. Denote the set of associated ideals of $M$ by $W(M)$.

In the following example we will show that there exist weakly primary submodules which are not primary.

EXAmple 5. Consider the $F[T]$-module $V$ of Example 4. We saw that (0) is not a primary submodule of $V$. We assert that (0) is a weakly primary submodule of $V$.

Suppose that $g(T) \in F[T]$ and there exists a nonzero vector $v \in V$ such that $g(T) v=0$. Now $g(T)$ has the form $f_{0}+f_{1} T+\cdots+f_{n} T^{n}$ where $f_{i} \in F$. It is evident that $f_{0}$ must be zero and so $g(T)=f_{1} T+f_{2} T^{2}+\cdots+f_{n} T^{n}$.

Let $x$ be any nonzero element of $V$. Then $x$ has the form $x=a_{1} v_{1}+a_{2} v_{2}+\cdots$ $+a_{k} v_{k}$ where $a_{i} \in F$ and $a_{k} \neq 0$. Wherefore $[g(T)]^{k} x=0$. Therefore (0) is a weakly primary submodule of $V$.

Proposition 10.1. Suppose that $R$ is a commutative ring and $M$ a finitely generated $R$-module. Then (0) is a primary submodule of $M$ if and only if $(0)$ is a weakly primary submodule of $M$. 
Proof. It is apparent that every primary submodule of $M$ is weakly primary.

Assume that (0) is a weakly primary submodule of $M$. Suppose that $r \in R$ annihilates a nonzero submodule of $M$. Then $r \in w(M)$. Let $m_{1}, \ldots, m_{k}$ be a set of generators for $M$. Since $r \in w(M)$, for each $i=1, \ldots, k$ there exists an integer $n_{i}$ such that $r^{n_{i}} m_{i}=0$. Hence there exists an integer $n$ such that $r^{n} m_{i}=0$ for each $i$, $1 \leqq i \leqq k$. Therefore $r^{n} M=0$ and so $r \in p(M)$. Consequently (0) is primary.

We will proceed to show that for a module over a Noetherian ring, $W$-submodules are weakly primary.

Lemma 10.2. Let $R$ be a commutative Noetherian ring and $M$ an $R$-module. Then $w(M)=t(M)$.

Proof. Let $r \in R$ be in $w(M)$. Consider the submodule (0:r) of $M$. Suppose that $N$ is a nonzero submodule of $M$. Let $x \neq 0$ be in $N$. Since $r \in w(M)$, there is an integer $n$ such that $r^{n} x=0$ and $r^{n-1} x \neq 0$. Then $(0: r) \cap N \neq(0)$. Consequently $(0: r)$ is an essential submodule of $M$. Therefore $r \in t(M)$.

Let $r \in t(M)$ and $x \in M$. For each $n, n=1,2, \ldots$, consider the ideal

$$
I_{n}=\left\{s \in R: r^{n} s x=0\right\}
$$

in $R$. Since $R$ is Noetherian, we have for large $n, I_{n}=I_{n+1}=\cdots$. If $I_{n} \neq R$ then $r^{n} R x \neq 0$. There exists an essential submodule $E$ of $M$ such that $r E=0$ because $r \in t(M)$. Thence $E \cap r^{n} R x \neq(0)$. If $r^{n} s x \neq 0$ is in $E \cap r^{n} R x$, then $r\left(r^{n} s x\right)=0$. Whence $s \in I_{n+1}$ but $s \notin I_{n}$. This is a contradiction. Hence $I_{n}=R$ and so $r^{n+1} x=0$. Therefore $r \in w(M)$.

Proposition 10.3. Let $R$ be a commutative Noetherian ring and $M$ an $R$-module. Then the following are equivalent:

(a) (0) is a $W$-submodule of $M$;

(b) (0) is a tertiary submodule of $M$;

(c) (0) is a weakly primary submodule of $M$.

Proof. Since $w(M)=t(M)$, it is immediate that (b) and (c) are equivalent.

Moreover (0) is a $W$-submodule of $M$ if and only if (0) is a $T$-submodule of $M$. Therefore the equivalence of (a) and (b) follows from Theorem 5.3.

Suppose that $R$ is a Noetherian ring and $C$ an admissible family of $w$-worthy $R$-modules. From Proposition 6.2 we see that for each $M$ in $C$ the elements of $W(M)$ are prime ideals. Theorem 4.10 shows that $W$ is a decomposition theory on $C$. We call $W$ the weakly primary theory on $C$ because 10.3 shows that (0) is a weakly primary submodule of $M$ in $C$ if and only if (0) is a $W$-submodule of $M$. The following corollary of 4.10 extends the results of Bourbaki found in [1, pp. 142-146].

COROLlaRY 10.4. Let $R$ be a commutative Noetherian ring with an identity and $C$ an admissible family of unitary $w$-worthy $R$-modules. Then the weakly primary decomposition theory, $W$ exists on $C$.

We will now consider an example of a module over a commutative Noetherian 
ring with an identity which is $t$-worthy and hence $w$-worthy; however, it is neither Noetherian nor Artinian. Corollary 10.4 shows that it has the weakly primary decomposition theory.

EXAMPLE 6. Let $p$ be a prime integer. Then the $p$-adic numbers $Q_{p}$ are a module over the $p$-adic integers $Z_{p}$. Now $Z_{p}$ is a commutative Noetherian ring with an identity. It can be shown that $Q_{p}$ is neither Noetherian nor Artinian; however, every factor module of $Q_{p}$ is 1-dimensional over $Z_{p}$. From Proposition 5.6 we have that $Q_{p}$ is a $t$-worthy $Z_{p}$-module.

11. Applications. The following proposition can be proved in a manner similar to [8, Proposition 1.6].

Proposition 11.1. Let $\Gamma$ be a decomposition function on an admissible family $C$ and let $M$ be in $C$. Suppose that the submodule $N$ of $M$ has a $\Gamma$-decomposition in $M$ and $Y$ is a submodule of $M$ containing $N$. Then there exists a submodule $X$ of $M$ such that $N=X \cap Y$ and $\Gamma(M / X)=\Gamma(Y / N)$.

This proposition shows that if $\Gamma$ is a decomposition theory on an admissible family $C$ and $M$ is in $C$ with $N \subseteq Y \subseteq M$, then there exists a submodule $X$ of $M$ such that $N=X \cap Y$ and $\Gamma(M / X)=\Gamma(Y / N)$. Furthermore $r_{\Gamma}(M / X)=r_{\Gamma}(Y / N)$, where $r_{\Gamma}$ is the radical function that is obtained from $\Gamma$ in $\S 3$.

The following theorem [4, p. 200] of W. Krull is now classical: if $\mathscr{A}$ is an ideal in a commutative Noetherian ring $R$ and if $Y=\bigcap_{n=1}^{\infty} \mathscr{A}^{n}$, then $Y=\mathscr{A} Y$. The proof does not extend to noncommutative left Noetherian rings because it uses the primary decomposition theory.

We notice that $Y$ is the intersection of all ideals $Q$ in $R$ such that $\mathscr{A}$ is contained in the primary radical of $R / Q$. If we replace primary radical by $r_{\Gamma}$, where $\Gamma$ is a decomposition theory, then we obtain the following generalization:

THEOREM 11.2. Let $R$ be an arbitrary ring and $\Gamma$ a decomposition theory on an admissible family $C$ of $R$-modules such that if $M$ is in $C$ and $\mathscr{B}$ is in $\Gamma(M)$ then $\mathscr{B} \supseteq(0: M)$. Suppose that $\mathscr{A}$ is a left ideal in $R, M$ is in $C$, and that

$$
Y=\cap\left\{Q: Q \text { is a submodule of } M \text { and } \mathscr{A} \subseteq r_{\Gamma}(M / Q)\right\} \text {. }
$$

Then $\mathscr{A} Y=Y$.

Proof. Since each $\mathscr{B} \in \Gamma(M)$ contains $(0: M)$, we have $(0: M) \subseteq r_{\Gamma}(M)$. Then $\mathscr{A} \subseteq(\mathscr{A} Y: Y) \subseteq r_{\Gamma}(Y / \mathscr{A} Y)$. From Proposition 11.1, there exists a submodule $X$ of $M$ such that $\mathscr{A} Y=X \cap Y$ and $r_{\Gamma}(M / X)=r_{\Gamma}(Y / \mathscr{A} Y)$. Therefore $\mathscr{A} \subseteq r_{\Gamma}(M / X)$ and hence $Y \subseteq X$. Consequently $\mathscr{A} Y=Y$.

COROLLARY 11.3. Let $R$ be an arbitrary ring and $C$ an admissible family of $t$-worthy $R$-modules. Suppose that $\mathscr{A}$ is a left ideal in $R, M$ is in $C$, and that

$$
Y=\bigcap\{Q: Q \text { is a submodule of } M \text { and } \mathscr{A} \subseteq t(M / Q)\},
$$

then $\mathscr{A} Y=Y$. 
Proof. Theorem 5.4 shows that the tertiary function, $T$, is a decomposition theory on $C$. Moreover if $M \in C$ and $\mathscr{B} \in T(M)$ then $\mathscr{B} \supseteq(0: M)$. On an admissible family of $t$-worthy $R$-modules, $r_{T}=t$ by 5.2 . Therefore the corollary is proved.

In [6, Théorème 2.1] Lesieur and Croisot have proved the following corollary with the addition Assumption 1 on $M$ :

COROLlary 11.4. Suppose that $R$ is an arbitrary ring and that either $M$ is an Artinian $R$-module or a Noetherian R-module. If $\mathscr{A}$ is a left ideal in $R$ and if

$$
Y=\bigcap\{Q: Q \text { is a submodule of } M \text { and } \mathscr{A} \subseteq t(M / Q)\},
$$

then $\mathscr{A} Y=Y$.

Corollary 11.5 (KRULl). If $\mathscr{A}$ is an ideal in a commutative Noetherian ring $R$ and if $Y=\bigcap_{n=1}^{\infty} \mathscr{A}^{n}$, then $Y=\mathscr{A} Y$.

Proof. Lemma 9.2 shows that for each ideal $I$ in $R, p(I)=t(I)$. Thus it is obvious that $\bigcap\{I: I$ is an ideal in $R$ and $\mathscr{A} \subseteq t(R / I)\} \subseteq Y$. Let $I$ be any ideal in $R$ such that $\mathscr{A} \subseteq t(R / I)$. Then $\mathscr{A} \subseteq p(R / I)$ and since $R$ is Noetherian there exists a positive integer $k$ such that $\mathscr{A}^{k} \subseteq I$. Therefore these two intersections are equal and so $Y=\mathscr{A} Y$.

Proposition 11.6. Let $R$ be a left Noetherian ring and $\Gamma$ a decomposition theory on an admissible family $C$ of $R$-modules such that if $M$ is in $C$ and $\mathscr{B}$ is in $\Gamma(M)$ then $\mathscr{B} \supseteq(0: M)$. Suppose that $\mathscr{A}$ is a nil left ideal in $R$. If

$$
Y=\bigcap\left\{Q: Q \text { is a submodule of } M \text { and } \mathscr{A} \subseteq r_{\Gamma}(M / Q)\right\},
$$

then $Y=(0)$.

Proof. By Levitzki's theorem [4, p. 199], there exists a positive integer $n$ such that $\mathscr{A}^{n}=(0)$. Theorem 11.2 shows that $\mathscr{A}^{n} Y=Y$. Therefore $Y=(0)$.

Proposition 11.7. Let $R$ be an arbitrary ring and $\Gamma$ a decomposition theory on an admissible family $C$ of Noetherian R-modules such that if $M$ is in $C$ and $\mathscr{B}$ is in $\Gamma(M)$ then $\mathscr{B} \supseteq(0: M)$. Suppose that $\mathscr{A}$ is a radical ideal in $R$ and $M$ is in $C$. If $Y=\bigcap\left\{Q: Q\right.$ is a submodule of $M$ and $\left.\mathscr{A} \subseteq r_{\Gamma}(M / Q)\right\}$, then $Y=(0)$.

Proof. From 11.2 we have that $\mathscr{A} Y=Y$. If $Y \neq(0)$ then $\mathscr{A} Y \neq Y$ by [4, p. 200, Proposition 2]. Therefore $Y=(0)$.

The following result is known as the Lemma of Artin-Rees [6, p. 219]: If $R$ is a commutative Noetherian ring and $M$ is a finitely generated $R$-module, then for each submodule $Y$ of $M$, ideal $\mathscr{A}$ in $R$, and positive integer $n$, there exists a positive integer $h$ such that $\mathscr{A}^{n} M \cap Y \subseteq \mathscr{A}^{n} Y$. This lemma is not valid for a finitely generated module $M$ over a noncommutative left Noetherian ring. See [6, p. 220].

The lemma can be interpreted thusly. For each submodule $Y$ of $M$, ideal $\mathscr{A}$ in $R$, and submodule $N$ of $Y$ such that $\mathscr{A} \subseteq p(Y / N)$, there exists an integer $n$ such that $\mathscr{A}^{n} Y \subseteq N$. Then the submodule $X=\mathscr{A}^{n} M+N$ satisfies $X \cap Y=N$ and $\mathscr{A} \subseteq p(M / X)$. 
If we replace $p$ by $r_{\Gamma}$ where $\Gamma$ is a decomposition theory, then we obtain the following generalization:

THEOREM 11.8. Let $R$ be an arbitrary ring, $\Gamma$ a decomposition theory on an admissible family $C$ of $R$-modules, and $M$ in $C$. For each submodule $Y$ of $M$, left ideal $\mathscr{A}$ in $R$, the submodule $N$ of $Y$ such that $\mathscr{A} \subseteq r_{\Gamma}(Y / N)$, there exists a submodule $X$ of $M$ such that $X \cap Y=N$ and $\mathscr{A} \subseteq r_{\mathrm{r}}(M / X)$.

Proof. From 11.1 there exists a submodule $X$ of $M$ such that $X \cap Y=N$ and $r_{\Gamma}(Y / N)=r_{\Gamma}(M / X)$. Therefore $\mathscr{A} \subseteq r_{\Gamma}(M / X)$ and the result follows.

COROLlaRY 11.9. Let $R$ be an arbitrary ring, $C$ an admissible family of t-worthy $R$-modules, and $M$ in $C$. For each submodule $Y$ of $M$, left ideal $\mathscr{A}$ in $R$, and the submodule $N$ of $Y$ such that $\mathscr{A} \subseteq t(Y / N)$, there exists a submodule $X$ of $M$ such that $X \cap Y=N$ and $\mathscr{A} \subseteq t(M / X)$.

Proof. Theorem 5.4 shows that $T$ is a decomposition theory on $C$. Moreover $r_{T}=t$ on $C$ by 5.2 .

Lesieur and Croisot have proved the following corollary in [6, Théorème 3.1] with the additional Assumption 1 on $M$ :

Corollary 11.10. Suppose that $R$ is an arbitrary ring and that either $M$ is an Artinian R-module or a Noetherian R-module. For each submodule $Y$ of $M$, left ideal $\mathscr{A}$ in $R$, and submodule $N$ of $Y$ such that $\mathscr{A} \subseteq t(Y / N)$, there exists a submodule $X$ of $M$ such that $X \cap Y=N$ and $\mathscr{A} \subseteq t(M / X)$.

COROllary 11.11. The Lemma of Artin-Rees.

Proof. Suppose that $M$ is a finitely generated module over a commutative Noetherian ring, $Y$ a submodule of $M, \mathscr{A}$ an ideal in $R$, and $n$ a positive integer. Lemma 9.2 shows that $\mathscr{A} \subseteq p\left(Y / \mathscr{A}^{n} Y\right)=t\left(Y / \mathscr{A}^{n} Y\right)$. There exists a submodule $X$ of $M$ such that $X \cap Y=\mathscr{A}^{n} Y$ and $\mathscr{A} \subseteq t(M / X)=p(M / X)$. Since $R$ is Noetherian there exists a positive integer $h$ such that $\mathscr{A}^{h} M \subseteq X$. Therefore $\mathscr{A}^{h} M \cap Y \subseteq \mathscr{A}^{n} Y$.

Let $R$ be a ring and $M$ an $R$-module. Then $M$ is said to be an Artin-Rees module if it has the Artin-Rees property, i.e., for each submodule $Y$ of $M$, ideal $\mathscr{A}$ in $R$, and positive integer $n$, there exists a positive integer $h$ such that $\mathscr{A}^{n} M \cap Y \subseteq \mathscr{A}^{n} Y$.

As soon as one has the tertiary decomposition theory and the primary decomposition theory the following question arises: When are tertiary submodules of an $R$-module $M$ primary? In Example 5 we saw a submodule that was tertiary but not primary. Lemma 9.2 shows that if $R$ is commutative and $M$ is Noetherian, then tertiary submodules of $M$ are primary. The following proposition shows that if $R$ is arbitrary and $M$ is an Artin-Rees $R$-module, then tertiary submodules of $M$ are primary:

Proposition 11.12. If $M$ is an Artin-Rees R-module, then tertiary submodules of $M$ are primary. 
Proof. In order to show that tertiary submodules of $M$ are primary, it suffices to show that for each submodule $N$ of $M, t(M / N) \subseteq p(M / N)$. Suppose that $r \in t(M / N)$. Then there exists an essential submodule $E / N$ of $M / N$ such that $r \in(N: E)$. The Artin-Rees property of $M$ applied to $E,(N: E)$, and $n=1$, produces an $h$ such that $(N: E)^{h} M \cap E \subseteq(N: E) E \subseteq N$. Since $E / N$ is essential, we have that $(N: E)^{n} M \subseteq N$. Thence $(N: E) \subseteq p(M / N)$. Whence $r \in p(M / N)$.

THEOREM 11.13. Let $R$ be a left Noetherian ring and $C$ an admissible family of $t$-worthy $R$-modules. Suppose that $M$ is in $C$. Then $M$ is an Artin-Rees module if and only if the tertiary submodules of $M$ are primary.

Proof. The "only if" follows from 11.12.

Assume that tertiary submodules of $M$ are primary. Let $Y$ be a submodule of $M, \mathscr{A}$ an ideal in $R$, and $n$ a positive integer. Consider $\mathscr{A}^{n} Y$. Since $\mathscr{A}^{n} \subseteq\left(\mathscr{A}^{n} Y: Y\right)$, we have that $\mathscr{A}^{n} \subseteq \mathscr{B}$ for all $\mathscr{B} \in T\left(Y / \mathscr{A}^{n} Y\right)$. Since $T$ is a decomposition theory on $C$, Proposition 11.1 shows that there exists a submodule $X$ of $M$ such that

$$
X \cap Y=\mathscr{A}^{n} Y \text { and } T(M / X)=T\left(Y / \mathscr{A}^{n} Y\right) .
$$

Since $M / X \in C$, there is a tertiary decomposition $\left\{M_{1}, M_{2}, \ldots, M_{k}\right\}$ of $X$ in $M$ with, say, $T\left(M / M_{i}\right)=\left\{\mathscr{B}_{i}\right\}$ where $i=1,2, \ldots, k$ and $T(M / X)=\left\{\mathscr{B}_{1}, \mathscr{B}_{2}, \ldots, \mathscr{B}_{k}\right\}$. Because $T(M / X)=T\left(Y / \mathscr{A}^{n} Y\right)$ we have that $\mathscr{A}^{n} \subseteq \mathscr{B}_{i}$ for $i=1,2, \ldots, k$.

By assumption the tertiary submodules $M_{i}, i=1,2, \ldots, k$ are primary and hence $\mathscr{B}_{i}=t\left(M / M_{i}\right)=p\left(M / M_{i}\right)$ for $i=1,2, \ldots, k$. Whence, for each $i=1,2, \ldots, k$, $\mathscr{B}_{i}$ is a nil ideal modulo $\left(M_{i}: M\right)$. From Levitzki's theorem we have that for each $i$, $i=1,2, \ldots, k$, there exists an $h_{i}$ such that $\mathscr{B}_{i}{ }_{i} \subseteq\left(M_{i}: M\right)$. Therefore there is an $h$ such that $\mathscr{B}_{i}^{h} M \subseteq M_{i}$ for each $i, i=1,2, \ldots, k$. Thus $\mathscr{A}^{n h} M \subseteq M_{1} \cap M_{2} \cap \cdots \cap M_{k}=X$. So $\mathscr{A}^{\text {nh }} M \cap Y \subseteq X \cap Y=\mathscr{A}^{n} Y$. Ergo $M$ is an Artin-Rees module.

The following corollary [6, Théorème 3.3 , corollaire] is due to Lesieur and Croisot:

COROLlARY. If $M$ is a finitely generated module over a left Noetherian ring, then $M$ is an Artin-Rees module if and only if the tertiary submodules of $M$ are primary.

Lemma 9.2 shows that tertiary submodules of a finitely generated module over a commutative Noetherian ring are primary. Hence the Lemma of Artin-Rees is also a corollary of 11.13 .

ACKNOWLedgment. The author wishes to express his deepest appreciation to Professor E. C. Dade for his helpful advice and encouragement during the preparation of this dissertation.

\section{REFERENCES}

1. N. Bourbaki, Éléments de mathématique. Fascicule XXVII. Algèbre commutative, Actualités Sci. Indust. No. 1293, Hermann, Paris, 1961.

2. A. W. Goldie, Rings with maximum condition, Yale Univ. Lecture Notes, New Haven, Conn., 1964. 
3. A. W. Goldie, Torsion-free modules and rings, J. Algebra 1 (1964), 268-287.

4. N. Jacobson, Structure of rings, Amer. Math. Soc. Colloq. Publ., Vol. 37, Amer. Math. Soc., Providence, R. I., 1956.

5. L. Lesieur and R. Croisot, Théorie noethérienne des anneaux, Math. Ann. 134 (1958), $458-476$

6. - Extension au cas non commutatif d'un théorème de Krull et d'un lemme d'Artin-Rees, J. Reine Angew. Math. 204 (1960), 216-220.

7. - Algèbre noethérienne non-commutatif, Memor. Sci. Math., Fasc. CLIV, Paris, 1963.

8. John A. Riley, Axiomatic primary and tertiary decomposition theory, Trans. Amer. Math. Soc. 105 (1962), 177-201.

9. O. Zariski and P. Samuel, Commutative algebra, Vol. I, Van Nostrand, Princeton, N. J., 1958.

\section{UNIVERSITY OF ILLINOIS,} URBANA, IlLINOIS 